\title{
STRICHARTZ ESTIMATES FOR THE SCHRÖDINGER EQUATION FOR THE SUBLAPLACIAN ON COMPLEX SPHERES
}

\author{
VALENTINA CASARINO AND MARCO M. PELOSO
}

\begin{abstract}
In this paper we consider the sublaplacian $\mathcal{L}$ on the unit complex sphere $S^{2 n+1} \subset \mathbf{C}^{n+1}$, equipped with its natural $\mathrm{CR}$ structure, and derive Strichartz estimates with fractional loss of derivatives for the solutions of the free Schrödinger equation associated with $\mathcal{L}$. Our results are stated in terms of certain Sobolev-type spaces that measure the regularity of functions on $S^{2 n+1}$ differently according to their spectral localization. Stronger conclusions are obtained for particular classes of solutions, corresponding to initial data whose spectrum is contained in a proper cone of $\mathbf{N}^{2}$.
\end{abstract}

\section{Introduction AND STATEMENT OF the MAIN RESUlts}

In the last two decades the dispersive properties of evolution equations have been extensively investigated and successfully applied in different contexts, such as the local and global existence for non-linear equations, the well-posedness theory in Sobolev spaces and the scattering theory. Dispersive and smoothing properties are now essentially well understood for the Schrödinger equation in the Euclidean setting, where both $L^{p}-L^{q}$ bounds and Strichartz estimates have been proved for a wide class of linear and non-linear problems.

In this paper, we study the dispersive properties of the free Schrödinger equation associated with the sublaplacian $\mathcal{L}$ on the unit complex sphere $S^{2 n+1}$ in $\mathbf{C}^{n+1}$, $n \geq 1$ :

$$
\left\{\begin{array}{l}
i \partial_{t} v+\mathcal{L} v=0 \\
v(0, z)=v_{0},
\end{array}\right.
$$

where $v_{0} \in L^{2}\left(S^{2 n+1}\right)$ and $\mathcal{L}$ denotes the sublaplacian, that is, the operator defined by

$$
\mathcal{L}:=-\sum_{1 \leq j<k \leq n+1} M_{j k} \bar{M}_{j k}+\bar{M}_{j k} M_{j k}
$$

with $M_{j k}:=\bar{z}_{j} \partial_{z_{k}}-\bar{z}_{k} \partial_{z_{j}}$. The operator $\mathcal{L}$ is a densely defined, self-adjoint, positive, and subelliptic operator on $S^{2 n+1}$ Ge] and it coincides with the real part of the Kohn-Laplacian acting on functions [Lee; see also MPR. The sublaplacian $\mathcal{L}$ may be considered as the subriemannian analogue of the Laplace-Beltrami operator on a Riemannian manifold; see e.g. JeLee.

Our main result is a Strichartz estimate for the solution $v$ of (1.1).

Received by the editors January 25, 2013.

2010 Mathematics Subject Classification. Primary 35Q41; Secondary 43A85, 35B65, 33C55.

Key words and phrases. Schrödinger equation, Strichartz estimates, complex spheres, sublaplacian, dispersive estimates. 
Strichartz estimates are a family of space-time bounds on solutions of (1.1), which provide a useful tool to control the norm of the solutions. In particular, using the notation $L_{t}^{p} L_{x}^{q}:=L^{p}\left(I_{t}, L^{q}\left(\mathbf{R}_{x}^{n}\right)\right)$, we bound the $L_{t}^{p} L_{x}^{q}$ norm of $v$ by means of a suitable mixed Sobolev norm, denoted by $\left\|v_{0}\right\|_{\mathcal{X}^{(r, s)}}$, of the initial datum.

In order to describe the mixed Sobolev spaces $\mathcal{X}^{(r, s)}$, we start from the classical decomposition of the space of square integrable functions on $S^{2 n+1}$,

$$
L^{2}\left(S^{2 n+1}\right)=\bigoplus_{\ell, \ell^{\prime}=0}^{\infty} \mathcal{H}^{\ell, \ell^{\prime}},
$$

with $\mathcal{H}^{\ell, \ell^{\prime}}$ being the space of complex spherical harmonics of bidegree $\left(\ell, \ell^{\prime}\right)$ ViK, Ch. 11].

This decomposition is the joint spectral decomposition of $\mathcal{L}$ and the LaplaceBeltrami operator $\Delta$ on $S^{2 n+1}$, since the subspaces $\mathcal{H}^{\ell, \ell^{\prime}}$ are eigenspaces both for $\Delta$ with eigenvalue $\mu_{\ell, \ell^{\prime}}=\left(\ell+\ell^{\prime}\right)\left(\ell+\ell^{\prime}+2 n\right)$, and for $\mathcal{L}$ with eigenvalue $\lambda_{\ell, \ell^{\prime}}=2 \ell \ell^{\prime}+n\left(\ell+\ell^{\prime}\right)$.

Now fix $M>1$ and define

$$
\mathcal{V}=\left\{\left(\ell, \ell^{\prime}\right) \in \mathbf{N}^{2}: \ell / M<\ell^{\prime}<M \ell\right\},
$$

and $\mathcal{E}$ to be its complementary region in $\mathbf{N}^{2}$. Observe that when $\left(\ell, \ell^{\prime}\right) \in \mathcal{V}$, then $\mu_{\ell, \ell^{\prime}} \approx \lambda_{\ell, \ell^{\prime}}$, while if $\left(\ell, \ell^{\prime}\right) \in \mathcal{E}$, that is, if $\ell^{\prime} \leq \ell / M$ or $M \ell \leq \ell^{\prime}$, then $\mu_{\ell, \ell^{\prime}}$ grows as $\max \left(\ell, \ell^{\prime}\right)^{2}$, while $\lambda_{\ell, \ell^{\prime}}$ varies between $\max \left(\ell, \ell^{\prime}\right)$ and $\max \left(\ell, \ell^{\prime}\right)^{2}$.

Hence, we are led to introduce appropriate Sobolev-type spaces that measure the regularity of functions differently according to their spectral localization. For $r \geq 0$ we denote by $W^{r}\left(S^{2 n+1}\right)$ the standard non-isotropic Sobolev space, for instance defined as the image of $L^{2}\left(S^{2 n+1}\right)$ under $(I+\mathcal{L})^{-r / 2}$.

We now define $\mathcal{X}_{M}^{(r, s)}\left(S^{2 n+1}\right)$ as the space of all functions $u \in L^{2}\left(S^{2 n+1}\right)$, spectrally decomposed as $u=\sum_{\ell, \ell^{\prime}=0}^{\infty} u_{\ell, \ell^{\prime}}, u_{\ell, \ell^{\prime}} \in \mathcal{H}^{\ell, \ell^{\prime}}$, such that

$$
\sum_{\ell / M<\ell^{\prime}<M \ell} u_{\ell, \ell^{\prime}} \in W^{r}\left(S^{2 n+1}\right),
$$

while the complementary sums

$$
\sum_{\ell^{\prime} \leq \ell / M} u_{\ell, \ell^{\prime}}, \sum_{\ell^{\prime} \geq M \ell} u_{\ell, \ell^{\prime}} \in W^{s}\left(S^{2 n+1}\right) .
$$

It may be seen as a natural fact that we need to consider a two-parameter scale for the Sobolev spaces, since (1.3) is a two-indices decomposition of $L^{2}\left(S^{2 n+1}\right)$.

The Strichartz estimates that we are able to prove for solutions of (1.1) are expressed in terms of $\mathcal{X}_{M}^{(r, s)}$ norms and are derived as a consequence of some kind of dispersive estimates. We denote by $Q:=2 n+2$ the homogeneous dimension of $S^{2 n+1}$ (see also Section 2).

Theorem 1.1. Let $S^{2 n+1}$ denote the unit complex sphere in $\mathbf{C}^{n+1}$ and let $\mathcal{L}$ be the sublaplacian, defined by (1.2). Let $p \geq 2, q<+\infty$ satisfy the admissibility condition

$$
\frac{2}{p}+\frac{Q}{q}=\frac{Q}{2}
$$


Define

$$
s_{n}:= \begin{cases}2[1-1 /(n+1)], & \text { if } n>1 \\ 4 / 3, & \text { if } n=1 .\end{cases}
$$

Let $M>1$ be fixed. Then, if $I$ is any finite time interval and $s \geq s_{1}$ or $s>s_{n}$ for $n>1$, there exists a constant $C=C(s, I, M)>0$ such that any solution $v$ of (1.1) satisfies the estimate

$$
\|v\|_{L^{p}\left(I, L^{q}\left(S^{2 n+1}\right)\right)} \leq C\left\|v_{0}\right\|_{\mathcal{X}_{M}^{(s / p, 2 / p)}\left(S^{2 n+1}\right)} .
$$

While there exists a vast literature on Strichartz estimates and their application to the non-linear Schrödinger equation for the Laplace-Beltrami operator on Riemannian manifolds (see the comments below), little is known in the case of the sublaplacian on CR manifolds, even in the case of the Heisenberg group $\mathbf{H}_{n}$. Indeed, since the Heisenberg group $\mathbf{H}_{n}$ is biholomorphically equivalent to the unit sphere $S^{2 n+1}$ with the north pole removed via the Cayley transform, Theorem 1.1 should be in particular compared with results concerning the Schrödinger equation on $\mathbf{H}_{n}$.

H. Bahouri, P. Gérard and C.-J. Xu in the seminal paper BaGX prove that no global in time dispersive estimate may hold for solutions of the Schrödinger equation on $\mathbf{H}_{n}$; see also the more recent work by Gérard and S. Grellier GG1, GG2. However, the same lack of dispersion occurs on $S^{2 n+1}$, and, in addition, no local in time dispersive estimate can hold, as we shall observe in Section 3 . Nonetheless, we are able to prove local Strichartz estimates for the solutions of (1.1) by substituting the dispersive estimate for the Schrödinger propagator $e^{i t \mathcal{L}}$ by a family of dispersive estimates for the frequency localized operator $e^{i t \mathcal{L}} \varphi\left(h^{2} \mathcal{L}\right)$. This idea originally appeared in $\mathrm{BaCh}$ and [Tat, and has been successfully applied in the work of Burq, Gérard and Tzvetkov BuGT1, BuGT2. In Theorem 3.1] we prove such spectrally localized dispersive estimates by means of a careful analysis of the oscillation of the infinite sum, depending on the two indices $\ell$ and $\ell^{\prime}$, that defines the integral kernel of the operator $e^{i t \mathcal{L}} \varphi\left(h^{2} \mathcal{L}\right)$. The proof of Theorem 3.1 is quite delicate and occupies a good portion of the present paper. One might wonder if the same technique could apply to the Heisenberg framework, and this topic will be the object of further investigation.

It is interesting to compare our results with the known ones in the Riemannian framework. Consider a Riemannian manifold $(\mathcal{M}, g)$ of dimension $d$ and the Schrödinger equation

$$
\left\{\begin{array}{l}
i \partial_{t} u+\Delta_{g} u=0 \\
u(0, x)=u_{0}
\end{array}\right.
$$

where $\Delta_{g}$ denotes the Laplace-Beltrami operator on $(\mathcal{M}, g)$. Then Strichartz estimates of solutions of (1.8) are usually of the form

$$
\|u\|_{L^{p}\left([-T, T], L^{q}(\mathcal{M})\right)} \leq C\left\|u_{0}\right\|_{H^{s}(\mathcal{M})},
$$

where $p, q, d$ satisy the scale-invariance condition

$$
\frac{2}{p}+\frac{d}{q}=\frac{d}{2} .
$$

Here and in what follows, we denote by $H^{s}$ the classical Sobolev space on $\mathcal{M}$, which may be defined as the image of $L^{2}(\mathcal{M})$ under $\left(I+\Delta_{g}\right)^{-s / 2}$. The key ingredient 
to prove (1.9) is given by some dispersive estimates, that is, estimates of the $L^{\infty}$ norm of solutions of (1.8) at a fixed time $t$.

When $\mathcal{M}=\mathbf{R}^{n}$, the theory is basically well established and one can choose $s=0$ and $T=\infty$ in (1.9), thanks to the essential contributions by Strichartz, Ginibre and Velo, and Keel and Tao Str, GiV, KT.

When $\mathcal{M}$ is a generic Riemannian manifold, the situation is more involved and the geometry, as it is well known, plays an essential rôle.

On compact manifolds the dispersive effect is generally weak. Nonetheless, Burq, Gérard and Tzvetkov, generalizing the earlier work of J. Bourgain on tori Bou1, Bou2, proved an estimate like (1.9) on any compact and boundaryless manifold $\mathcal{M}$, with $s=1 / p$, with a loss of derivatives with respect to the flat Euclidean case, but again with a gain of $1 / p$ derivatives in comparison to the bounds indicated by Sobolev embeddings BuGT2. Later, Blair, Smith and Sogge proved Strichartz estimates with $s=4 / 3 p$ both for a compact Riemannian manifold with boundary and for a compact manifold $\mathcal{M}$ without boundary, endowed with a Lipschitz metric $g$ BlSSo1] (see also BlSSo2, where these results have been recently improved).

Then the spirit of Theorem 1.1 is that if the initial datum $v_{0}$ is spectrally localized in a proper angular sector $\mathcal{V}$ in $\mathbf{N}^{2}$ defined as in (1.4), then we are able to prove a Strichartz estimate like (1.9), where the $H^{s}$ norm of the initial datum at the right-hand side is replaced by the standard non-isotropic norm $W^{s / p}$, for any index $s$ such that $s>2[1-1 /(n+1)]$ if $n>1$ or $s \geq 4 / 3$ if $n=1$. Thus there is a gain of $2 /(n+1) p$ derivatives $(2 / 3 p$ in the one-dimensional case) in comparison to the bounds indicated by non-isotropic Sobolev embeddings. If the initial datum $v_{0}$ is spectrally localized in the complementary region, that is, for instance, if $v_{0}=$ $\sum_{0 \leq \ell^{\prime} \leq \ell / M} h_{\ell, \ell^{\prime}}, h_{\ell, \ell^{\prime}} \in \mathcal{H}^{\ell, \ell^{\prime}}$, then our techniques only lead to an estimate like (1.9), with the $H^{s}$ norm of the initial datum at the right-hand side replaced by the standard non-isotropic norm $W^{2 / p}$, thus providing no improvement with respect to the Sobolev embedding.

The problem of optimality for Strichartz estimates is in general open, also in the Riemannian set-up. As it is well known, the Strichartz estimate proved in BuGT2 is not sharp, unless in the case $p=2$, in the class of compact Riemannian manifolds, since J. Bourgain proved that for the flat torus $(\mathbf{R} / 2 \pi \mathbf{Z})^{2}$ the Strichartz estimate holds for $p=q=2$ with loss of $\varepsilon$ derivatives, for every $\varepsilon>0$ [Bou1, Bou2]. Moreover, Burq, Gérard and Tzvetkov were able to improve their intermediate Strichartz estimates in some specific geometries, like spheres and Zoll surfaces BuGT3, BuGT4. In our framework, some sharp bounds for the eigenfunctions of the sublaplacian on $S^{2 n+1}$, recently proved by the first author [Ca1, Ca2, do not suffice to prove the optimality in (1.7).

It is worth noticing that different approaches, which have been succesfully used in the Riemannian context (we refer in particular to BuGT3, BuGT2]), are possible and could be used to prove optimal bounds, at least for intermediate $(p, q)$. In particular, it would be interesting to prove multilinear estimates for spectral projections associated to $\mathcal{L}$ on the complex sphere, as well as to prove intermediate Strichartz estimates by following the Fourier analytic approach by Bourgain.

We would also like to point out that the compact manifold $S^{2 n+1}$, beyond the pioneering works of G. Folland and D. Geller [Fo1, Ge], has recently attracted a lot of interest in connection with its CR structure; we refer, in particular, to the recent papers $\mathrm{BrFM}, \mathrm{BauW}, \mathrm{CowKS}$ and to $\mathrm{Ca1}, \mathrm{Ca} 2, \mathrm{CaP} 1]$. 
The paper is organized as follows. In Section 2 we start recalling the basic facts about harmonic analysis on the complex sphere. Then we recall the definition of the standard isotropic and non-isotropic Sobolev spaces on the sphere and introduce the mixed Sobolev spaces. In Sections 3 and 4 we prove the basic dispersive estimates for solutions of (1.1) localized at high frequencies. Our proof hinges on a repeated use of the Poisson summation formula, specifically adapted in the key Lemma 4.4 to our case. Following a classical approach, we then deduce in Section 5 the Strichartz estimate (1.7) from the dispersive bounds. Optimality will be discussed in Section 6. where we also make a comment on other possible admissibility conditions.

We shall use the symbol $C$ to denote constants which may vary from one formula to the next, and $\lfloor x\rfloor$ to denote the greatest integer at most $x$. The symbol $\approx$ between two positive expressions means that their ratio is bounded above and below.

\section{Preliminary faCts AND NOtation}

In this section we recall some basic facts about spherical harmonics and their relation to the the analysis on the complex sphere.

For $n \geq 1$, we denote by $\mathbf{C}^{n+1}$ the $(n+1)$-dimensional complex space equipped with the scalar product $\langle z, w\rangle:=z_{1} \bar{w}_{1}+\cdots+z_{n+1} \bar{w}_{n+1}, z, w \in \mathbf{C}^{n+1}$, and by $S^{2 n+1}$ the unit sphere in $\mathbf{C}^{n+1}$,

$$
S^{2 n+1}=\left\{z=\left(z_{1}, \ldots, z_{n+1}\right) \in \mathbf{C}^{n+1}:\langle z, z\rangle=1\right\} .
$$

The sphere $S^{2 n+1}$ is a strongly pseudoconvex CR manifold and thus endowed with subriemannian structure. The Carnot-Carathéodory distance associated with the operator $\mathcal{L}$ is equivalent to the so-called Korányi distance $d$,

$$
d(z, w):=|1-\langle z, w\rangle|^{1 / 2},
$$

$z, w \in S^{2 n+1} ;$ see $[\mathrm{Na}]$.

The homogeneous dimension $Q$ of $S^{2 n+1}$, that will play a relevant role in our analysis, is given by $Q:=2 n+2$, since it is well known that $\operatorname{Vol}(B(z, r)) \sim r^{Q}$, where $B(z, r)$ denotes the ball centered at $z \in S^{2 n+1}$ with radius $r>0$.

2.1. Spherical harmonics and spectral projections. Consider the space $L^{2}\left(S^{2 n+1}\right)$, equipped with the inner product

$$
(f, g):=\int_{S^{2 n+1}} f(z) \overline{g(z)} d \sigma(z),
$$

where $d \sigma$ is the Lebesgue surface measure, which is invariant under the action of the unitary group $U(n+1)$.

For non-negative integers $\ell, \ell^{\prime}, \mathcal{H}^{\ell, \ell^{\prime}}$ is the vector space of the restrictions to $S^{2 n+1}$ of harmonic polynomials $p(z, \bar{z})$, homogeneous of degree $\ell$ in $z$ and of degree $\ell^{\prime}$ in $\bar{z}$. A function in $\mathcal{H}^{\ell, \ell^{\prime}}$ is called a complex spherical harmonic of bidegree $\left(\ell, \ell^{\prime}\right)$.

When $\ell^{\prime}=0$, the space $\mathcal{H}^{\ell, 0}$ consists of holomorphic polynomials and $\mathcal{H}^{0, \ell}$ consists of polynomials whose complex conjugates are holomorphic.

The subspaces $\mathcal{H}^{\ell, \ell^{\prime}}$ have finite dimension $d_{\ell, \ell^{\prime}}$ given by

$$
d_{\ell, \ell^{\prime}}:=n \frac{\ell+\ell^{\prime}+n}{\ell \ell^{\prime}}\left(\begin{array}{c}
\ell+n-1 \\
\ell-1
\end{array}\right)\left(\begin{array}{c}
\ell^{\prime}+n-1 \\
\ell^{\prime}-1
\end{array}\right)
$$


if $\ell, \ell^{\prime} \geq 1$, and by

$$
d_{\ell, 0}=d_{0, \ell}:=\left(\begin{array}{c}
\ell+n \\
\ell
\end{array}\right)
$$

if $\ell$ or $\ell^{\prime}$ equals 0 .

Moreover, the subspaces $\mathcal{H}^{\ell, \ell^{\prime}}$ are $U(n+1)$-invariant, pairwise orthogonal and their sum is dense in $L^{2}\left(S^{2 n+1}\right)$; more explicitly, if we denote by the symbol $\pi_{\ell, \ell^{\prime}}$ the orthogonal projector mapping $L^{2}\left(S^{2 n+1}\right)$ onto $\mathcal{H}^{\ell, \ell^{\prime}}$, then each function $f \in$ $L^{2}\left(S^{2 n+1}\right)$ may be decomposed in a unique way as

$$
f=\sum_{\ell, \ell^{\prime}=0}^{+\infty} \pi_{\ell, \ell^{\prime}} f,
$$

where the series converges unconditionally to $f$ in the $L^{2}$-topology.

A special role in $\mathcal{H}^{\ell, \ell^{\prime}}$ is played by the so-called zonal functions. Let $\left\{Y_{k}^{\ell, \ell^{\prime}}\right\}$, $k=1, \ldots, d_{\ell, \ell^{\prime}}$, be an orthonormal basis for $\mathcal{H}^{\ell, \ell^{\prime}}$. For $(z, w) \in S^{2 n+1} \times S^{2 n+1}$ set

$$
Z_{\ell, \ell^{\prime}}(z, w):=\sum_{k=1}^{d_{\ell, \ell^{\prime}}} Y_{k}^{\ell, \ell^{\prime}}(z) \overline{Y_{k}^{\ell, \ell^{\prime}}(w)} .
$$

Then, for all $f \in \mathcal{H}^{\ell, \ell^{\prime}}$ we have

$$
f(z)=\int_{S^{2 n+1}} f(w) Z_{\ell, \ell^{\prime}}(z, w) d \sigma(w) .
$$

Since $\mathcal{H}^{\ell, \ell^{\prime}}$ is finite dimensional, the above pairing makes sense for all $f \in L^{2}\left(S^{2 n+1}\right)$.

For each fixed point $w \in S^{2 n+1}$, the function $f(w)=Z_{\ell, \ell^{\prime}}(\cdot, w)$ is in $\mathcal{H}^{\ell, \ell^{\prime}}$ and is constant on the orbits of the stabilizer of $w$ in $U(n+1)$, which is isomorphic to $U(n)$. In other words $Z_{\ell, \ell^{\prime}}(z, w)$ depends only on $\langle z, w\rangle$, and we write

$$
\langle z, w\rangle=e^{i \omega} \cos \theta, \quad \theta \in[0, \pi / 2], \omega \in[0,2 \pi) .
$$

With an abuse of notation, we will also denote by $Z_{\ell, \ell^{\prime}}$ the function depending on the 1-dimensional complex variable $\langle z, w\rangle$, that is,

$$
Z_{\ell, \ell^{\prime}}(\langle z, w\rangle)=Z_{\ell, \ell^{\prime}}(z, w) .
$$

An explicit formula for the zonal function $Z_{\ell, \ell^{\prime}} \in \mathcal{H}^{\ell, \ell^{\prime}}$, for $\ell^{\prime} \geq \ell \geq 1$, is given by

$$
Z_{\ell, \ell^{\prime}}\left(e^{i \omega} \cos \theta\right)=\frac{d_{\ell, \ell^{\prime}}}{\omega_{2 n+1}} \frac{\ell !(n-1) !}{(\ell+n-1) !} e^{i \omega\left(\ell^{\prime}-\ell\right)}(\cos \theta)^{\ell^{\prime}-\ell} P_{\ell}^{\left(n-1, \ell^{\prime}-\ell\right)}(\cos 2 \theta),
$$

where $\omega_{2 n+1}$ denotes the surface area of $S^{2 n+1}$ and $P_{\ell^{\prime}}^{\left(n-1, \ell-\ell^{\prime}\right)}$ is the Jacobi polynomial; see $\mathrm{Sz}]$.

For the case $\ell^{\prime}<\ell$, it suffices to recall that $Z_{\ell, \ell^{\prime}}(z, w)=\overline{Z_{\ell^{\prime}, \ell}(w, z)}$.

Since $P_{0}^{(n-1, \ell)} \equiv 1$, if $\ell^{\prime}=0$ the zonal function is given by

$$
Z_{\ell, 0}(z, w)=\frac{1}{\omega_{2 n+1}}\left(\begin{array}{c}
\ell+n \\
\ell
\end{array}\right) \overline{\langle z, w\rangle}^{\ell} .
$$

The following bound for the zonal functions is well known and appears in [Fo3]. For any $z, w \in S^{2 n+1}$ we have

$$
\left|Z_{\ell, \ell^{\prime}}(z, w)\right| \leq \frac{d_{\ell, \ell^{\prime}}}{\omega_{2 n+1}}
$$


Finally, it is easy to check that the orthogonal projector $\pi_{\ell, \ell^{\prime}}$ may be written as

$$
\pi_{\ell, \ell^{\prime}} f(z)=\int_{S^{2 n+1}} f(w) Z_{\ell, \ell^{\prime}}(z, w) d \sigma(w) .
$$

2.2. Classical and non-isotropic Sobolev spaces. Recall the decomposition (1.3) of $L^{2}\left(S^{2 n+1}\right)$. Each subspace $\mathcal{H}^{\ell, \ell^{\prime}}$ is an eigenspace both for Laplace-Beltrami operator $\Delta$ with eigenvalue $\mu_{\ell, \ell^{\prime}}:=\left(\ell+\ell^{\prime}\right)\left(\ell+\ell^{\prime}+2 n\right)$, and for $\mathcal{L}$ with eigenvalue $\lambda_{\ell, \ell^{\prime}}:=2 \ell \ell^{\prime}+n\left(\ell+\ell^{\prime}\right)$. For these and other properties of $\mathcal{L}$ we refer the reader to $\mathrm{Ge}$ and $\mathrm{RU}$.

The non-isotropic Sobolev spaces on the complex sphere can be defined in terms of suitable powers of $I+\mathcal{L}$ or, equivalently, in terms of suitable powers of the conformal sublaplacian $\mathcal{D}:=\mathcal{L}+\frac{n^{2}}{2}$; see, for instance, [Fo2]. More precisely, for $1 \leq p \leq \infty$ we set

$$
W^{r, p}\left(S^{2 n+1}\right):=\left\{f \in L^{p}\left(S^{2 n+1}\right):(I+\mathcal{L})^{r / 2} f \in L^{p}\right\} .
$$

The operator $(I+\mathcal{L})^{r / 2}$ can be defined locally transferring the analogous operator from the Heisenberg group via the Cayley transform; see [Fo2, § 3].

We will mostly deal with the case of $L^{2}$-integrability, and we simply write $W^{r}$ for $W^{r, 2}$. For functions in $W^{r}$ we have the identity

$$
(I+\mathcal{L})^{r / 2} f=\sum_{\ell, \ell^{\prime}=0}^{+\infty}\left(1+\lambda_{\ell, \ell^{\prime}}\right)^{r / 2} \pi_{\ell, \ell^{\prime}} f .
$$

Then, $W^{r}$ is a Hilbert space under the inner product

$$
(f, g)_{W^{r}}:=\int_{S^{2 n+1}}(I+\mathcal{L})^{r / 2} f \overline{(I+\mathcal{L})^{r / 2} g} .
$$

For $s \geq 0$, we shall denote by $H^{s}\left(S^{2 n+1}\right)$ the classical Sobolev space on $S^{2 n+1}$, defined as in (2.8), with the operator $I+\mathcal{L}$ replaced by the operator $I+\Delta$. In particular, $H^{s}$ is endowed with the norm

$$
\|f\|_{H^{s}}=\left(\sum_{\ell, \ell^{\prime}=0}^{\infty}\left(1+\mu_{\ell, \ell^{\prime}}\right)^{s}\left\|\pi_{\ell, \ell^{\prime}} f\right\|_{L^{2}}^{2}\right)^{1 / 2} .
$$

The following inclusions follow:

$$
H^{s} \subseteq W^{s} \subseteq H^{s / 2} .
$$

For both isotropic and non-isotropic Sobolev immersion theorems in a CR setting we refer to the seminal papers $\mathrm{Fo} 2$ and $\mathrm{FoSt}$, where results are proved in the framework of Heisenberg groups. Anyway, it is not difficult to check that the same inclusions hold on complex spheres.

2.3. Mixed Sobolev spaces. We now introduce a family of Sobolev-type spaces that measure the regularity of functions differently according to their spectral localization.

Fix a constant $M>1$ and define the proper cone $\mathcal{V}=\mathcal{V}_{M}$ in $\mathbf{N}^{2}$,

$$
\mathcal{V}:=\left\{\left(\ell, \ell^{\prime}\right): \ell / M<\ell^{\prime}<M \ell\right\},
$$

and the pair of edges $\mathcal{E}=\mathcal{E}_{M}$

$$
\mathcal{E}:=\left\{\left(\ell, \ell^{\prime}\right): \ell^{\prime} \leq \ell / M \text { or } \ell^{\prime} \geq M \ell\right\} .
$$


We define the corresponding spectral projections

$$
\pi_{\mathcal{V}}=\sum_{\ell / M<\ell^{\prime}<M \ell} \pi_{\ell, \ell^{\prime}} \text { and } \pi_{\mathcal{E}}=\sum_{\ell^{\prime} \leq \ell / M \text { or } \ell^{\prime} \geq M \ell} \pi_{\ell, \ell^{\prime}}
$$

We then introduce the corresponding spaces of spectrally localized functions:

$$
L_{\mathcal{V}}^{2}\left(S^{2 n+1}\right)=\left\{u \in L^{2}\left(S^{2 n+1}\right): u=\pi_{\mathcal{V}} u\right\}
$$

and

$$
L_{\mathcal{E}}^{2}\left(S^{2 n+1}\right)=\left\{u \in L^{2}\left(S^{2 n+1}\right): u=\pi_{\mathcal{E}} u\right\} .
$$

We define the mixed Sobolev spaces $\mathcal{X}^{(r, s)}=\mathcal{X}_{M}^{(r, s)}\left(S^{2 n+1}\right)$ as

$$
\mathcal{X}^{(r, s)}=\left\{u \in L^{2}\left(S^{2 n+1}\right): \pi_{\mathcal{V}} u \in W^{r}\left(S^{2 n+1}\right) \text { and } \pi_{\mathcal{E}} u \in W^{s}\left(S^{2 n+1}\right)\right\},
$$

with norm given by

$$
\|u\|_{\mathcal{X}(r, s)}=\left(\sum_{\left(\ell, \ell^{\prime}\right) \in \mathcal{V}}\left(1+\lambda_{\ell, \ell^{\prime}}\right)^{r}\left\|\pi_{\ell, \ell^{\prime}} u\right\|_{L^{2}}^{2}+\sum_{\left(\ell, \ell^{\prime}\right) \in \mathcal{E}}\left(1+\lambda_{\ell, \ell^{\prime}}\right)^{s}\left\|\pi_{\ell, \ell^{\prime}} u\right\|_{L^{2}}^{2}\right)^{1 / 2} .
$$

Notice that the norm depends on $M$ although it will not be explicitly indicated.

In general, given a function space $\mathcal{Y} \subseteq L^{2}\left(S^{2 n+1}\right)$, we denote by $\mathcal{Y}_{\mathcal{V}}$ and $\mathcal{Y}_{\mathcal{E}}$ respectively, the subspaces of $\mathcal{Y}$ of the functions that are spectrally localized in $\mathcal{V}$ and $\mathcal{E}$, respectively. Then, we have

$$
\mathcal{X}^{(r, s)}=W_{\mathcal{V}}^{r} \cap W_{\mathcal{E}}^{s} .
$$

For the mixed Sobolev spaces $\mathcal{X}^{(r, s)}$ we have the following elementary result that gives embedding in the Lebesgue spaces and comparison with the classical non-isotropic Sobolev spaces.

Proposition 2.1. Let $M>1$ be fixed. Given $r, s \geq 0$ the following properties hold true:

(1) If $\min (r, s)>Q\left(\frac{1}{2}-\frac{1}{q}\right)$, then for all $u \in \mathcal{C}^{\infty}\left(S^{2 n+1}\right)$ we have

$$
\|u\|_{L^{q}} \leq C\|u\|_{\mathcal{X}^{(r, s)}} .
$$

(2) If $u \in \mathcal{C}_{\mathcal{V}}^{\infty}$, then

$$
\begin{gathered}
\|u\|_{\mathcal{X}^{(r, s)}} \approx\|u\|_{W^{r}} \approx\|u\|_{H^{r}}, \\
\text { and, for } \min (r, s)>(2 n+1)\left(\frac{1}{2}-\frac{1}{q}\right), \\
\|u\|_{L^{q}} \leq C\|u\|_{\mathcal{X}^{(r, s)}} .
\end{gathered}
$$

(3) For all $u \in \mathcal{C}^{\infty}$ such that $\pi_{\ell, \ell^{\prime}} u=0$ for $\min \left(\ell, \ell^{\prime}\right)>M$, we have

$$
\|u\|_{\mathcal{X}^{(r, s)}}=\|u\|_{W^{r}} \approx\|u\|_{H^{r / 2}} .
$$

The constants involved in the above estimates depend on $M$.

Proof. (1) It suffices to recall the embedding theorems for the non-isotropic Sobolev spaces $W^{r}$. Theorem 5.15 in Fo2 entails that if $u \in \mathcal{C}_{\mathcal{V}}^{\infty}$, then $\|u\|_{L^{q}} \leq C\|u\|_{W^{r, 2}}$ if $r>Q\left(\frac{1}{2}-\frac{1}{q}\right)$. The result now follows easily.

Next, for $u \in \mathcal{C}_{\mathcal{V}}^{\infty}$ we have

$$
\begin{aligned}
\|u\|_{(r, s)}^{2} & \approx \sum_{\ell / M<\ell^{\prime}<M \ell}\left(1+\lambda_{\ell, \ell^{\prime}}\right)^{r}\left\|\pi_{\ell, \ell^{\prime}} u\right\|_{2}^{2} \approx \sum_{\ell / M<\ell^{\prime}<M \ell}(1+\ell)^{2 r}\left\|\pi_{\ell, \ell^{\prime}} u\right\|_{2}^{2} \\
& \approx \sum_{\ell / M<\ell^{\prime}<M \ell}\left(1+\mu_{\ell, \ell^{\prime}}\right)^{r}\left\|\pi_{\ell, \ell^{\prime}} u\right\|_{2}^{2} .
\end{aligned}
$$


The second statement in (2) now follows from the classical embedding theorem for the Sobolev space $H^{\sigma}\left(S^{2 n+1}\right)$.

Finally, (3) follows at once since, for $\min \left(\ell, \ell^{\prime}\right) \leq M,\left(1+\lambda_{\ell, \ell^{\prime}}\right) \approx\left(1+\mu_{\ell, \ell^{\prime}}\right)^{1 / 2}$.

\section{The Dispersive EStimate}

In this section we study the dispersive properties for solutions of the Schrödinger equation that are spectrally localized.

The solutions of (1.1) do not satisfy in general a dispersive estimate, either globally (as constant solutions show for large $t$ ) or locally in time. A similar lack of the dispersive effect was noticed by Burq, Gérard and Tzvetkov in the Riemannian case on compact manifolds as well (see [BuGT2]).

Indeed, if we could prove a dispersive estimate of the form

$$
\| e^{i t \mathcal{L}_{\left(L^{1}\left(S^{2 n+1}\right), L^{\infty}\left(S^{2 n+1}\right)\right)}} \leq \frac{C}{|t|^{q_{0}}}
$$

for some $q_{0}>0$ and for some $t>0$, then the $L^{\infty}$ norm of eigenfunctions of the sublaplacian should be controlled by the $L^{1}$ norm, and this is not true in general (see Theorem 3.1 in $\mathrm{Ca} 2$, where bounds are proved for the $L^{1}$ norm of zonal functions).

However, it is possible to prove a family of dispersive estimates on small time intervals related to the frequencies of the data that will suffice for the proof of the Strichartz estimates (see [BaCh] and Tat for a first application of this idea).

In this paper, we are able to prove different dispersive estimates for data $v$ that are spectrally localized according to a double decomposition of the spectrum.

We introduce a spectral cut-off. Let $\varphi$ be a non-negative smooth function with support contained in the interval $[a, b]$, with $0 \leq a \leq b<\infty$. For $h \in(0,1]$, we consider the operator

$$
\varphi\left(h^{2} \mathcal{L}\right): L^{2}\left(S^{2 n+1}\right) \rightarrow L^{2}\left(S^{2 n+1}\right),
$$

defined by the functional calculus for the sublaplacian.

Next, we fix a smooth cut-off function $\psi$ with compact support in $[1 / M, M]$, where $M>1$ is a (large) constant.

Theorem 3.1. Let $\varphi, \psi$ be smooth cut-off functions defined as above. Let $p, p^{\prime}$ be such that $\frac{1}{p}+\frac{1}{p^{\prime}}=1, p \in[1,2]$. Then the following estimates hold:

(i) Let $s_{n}$ be as in (1.6) and let $s>s_{n}$ if $n>1$, or $s \geq s_{n}$ if $n=1$. Then there exist $c, C_{s}>0$ such that for all $v_{0} \in \mathcal{C}^{\infty}\left(S^{2 n+1}\right)$, for all $h \in(0,1]$,

$$
\begin{aligned}
& \left\|\sum_{\ell, \ell^{\prime} \geq 0} e^{i t \lambda_{\ell, \ell^{\prime}}} \varphi\left(h^{2} \lambda_{\ell, \ell^{\prime}}\right) \psi\left(\ell^{\prime} / \ell\right) \pi_{\ell, \ell^{\prime}}\left(v_{0}\right)\right\|_{L^{p^{\prime}\left(S^{2 n+1}\right)}} \leq \frac{C_{s}}{|t|^{Q / 2\left(\frac{1}{p}-\frac{1}{p^{\prime}}\right)}}\left\|v_{0}\right\|_{L^{p}\left(S^{2 n+1}\right)} \\
& \text { for all } t \in I_{s}:=\left[-c h^{s}, c h^{s}\right] \text {. }
\end{aligned}
$$

(ii) Then there exists $C>0$ such that for all $v_{0} \in \mathcal{C}^{\infty}\left(S^{2 n+1}\right)$, for all $h \in(0,1]$,

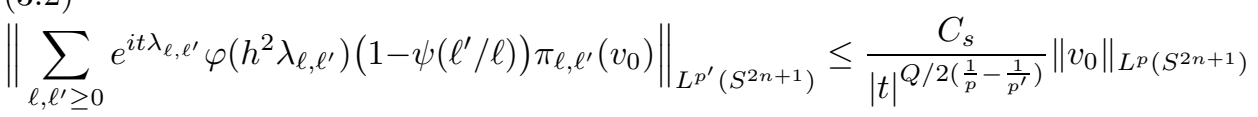

$$
\begin{aligned}
& \text { for all } t \in I_{2}:=\left[-h^{2}, h^{2}\right] \text {. }
\end{aligned}
$$


Remark 3.2. We point out that the index $s$ which determines the length of the time interval $I_{s}$ in (3.1) and (3.2) is subject to the following upper bound. For, the kernel of the operator $e^{i t \mathcal{L}} \varphi\left(h^{2} \mathcal{L}\right)$ is given by

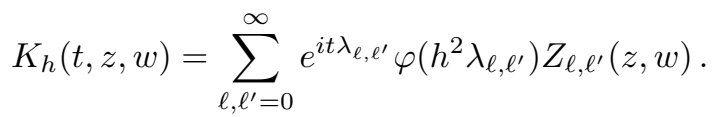

Reasoning as in BuGT2, we have

$$
\begin{aligned}
\left\|e^{i t \mathcal{L}} \varphi\left(h^{2} \mathcal{L}\right)\right\|_{\left(L^{1}, L^{\infty}\right)} & =\left\|K_{h}(t, \cdot, \cdot)\right\|_{L^{\infty}\left(S^{2 n+1} \times S^{2 n+1}\right)} \geq C\left\|K_{h}(t, \cdot, \cdot)\right\|_{L^{2}\left(S^{2 n+1} \times S^{2 n+1}\right)} \\
& \geq C\left(\sum_{\ell, \ell^{\prime}=0}^{\infty}\left|\varphi\left(h^{2} \lambda_{\ell, \ell^{\prime}}\right)\right|^{2} d_{\ell, \ell^{\prime}}\right)^{1 / 2} \geq \frac{C}{h^{n-1}}\left(\sum_{\lambda_{\ell, \ell^{\prime}} \sim h^{-2}}\left(\ell+\ell^{\prime}\right)\right)^{1 / 2} \\
& \geq \frac{C}{h^{n-1}}\left(\sum_{\ell \sim h^{-2}} \ell\right)^{1 / 2}=\frac{C}{h^{n+1}},
\end{aligned}
$$

where in particular we used (2.2). Then estimates like (3.1) or (3.2) for $p=1$ imply $|t| \leq c h$ for some $c>0$, that is, $s \geq 1$.

Proof of Theorem 3.1. For all $t \in \mathbf{R}$ we have

$$
\left\|e^{i t \mathcal{L}} \varphi\left(h^{2} \mathcal{L}\right) v_{0}\right\|_{L^{2}} \leq C\left\|v_{0}\right\|_{L^{2}}
$$

Thus, as a consequence of the Riesz-Thorin Theorem and Young's inequality, it suffices to prove the following estimates:

$$
\left\|\sum_{\ell, \ell^{\prime} \geq 0} e^{i t \lambda_{\ell, \ell^{\prime}}} \varphi\left(h^{2} \lambda_{\ell, \ell^{\prime}}\right) \psi\left(\ell^{\prime} / \ell\right) Z_{\ell, \ell^{\prime}}\right\|_{L^{\infty}\left(S^{2 n+1} \times S^{2 n+1}\right)} \leq \frac{C}{|t|^{Q / 2}}
$$

for all $|t| \leq h^{s}$, where $s>s_{n}:=2[1-1 /(n+1)]$ and

$$
\left\|\sum_{\ell, \ell^{\prime} \geq 0} e^{i t \lambda_{\ell, \ell^{\prime}}} \varphi\left(h^{2} \lambda_{\ell, \ell^{\prime}}\right)\left(1-\psi\left(\ell^{\prime} / \ell\right)\right) Z_{\ell, \ell^{\prime}}\right\|_{L^{\infty}\left(S^{2 n+1} \times S^{2 n+1}\right)} \leq \frac{C}{|t|^{Q / 2}}
$$

for all $|t| \leq h^{2}$.

In order to prove Theorem 3.1 we break the proof of (3.3) and (3.4) into a few steps, that now we summarize.

Step 1. We prove both estimates when (i) $h \geq \varepsilon_{0}$, where $\varepsilon_{0}>0$ is a fixed constant and (ii) when $|t| \leq h^{2}$. This second case proves in fact that both (3.3) and (3.4) hold for these values of $t$ and in particular establishes (3.2) in Theorem 3.1.

Step 2. Recalling (2.6) and (2.5), we prove (3.3) when $\langle z, w\rangle=e^{i \omega} \cos \theta$ varies in a fixed compact set of the unit disk, that is, when $\theta \in\left[\varepsilon_{1}, \pi / 2\right]$, for some $\varepsilon_{1}>0$.

Step 3. Next, we assume that $h^{2} \leq|t| \leq h^{s}$ and $0<h<\varepsilon_{0}$, and prepare to estimate

$$
\left|\sum_{\ell, \ell^{\prime}=1}^{+\infty} e^{i t \lambda_{\ell, \ell^{\prime}}} \varphi\left(h^{2} \lambda_{\ell, \ell^{\prime}}\right) \psi\left(\ell^{\prime} / \ell\right) Z_{\ell, \ell^{\prime}}(z, w)\right|
$$

when $\langle z, w\rangle$ varies outside the compact set fixed in Step 2, that is, when $\theta<\varepsilon_{1}$.

First we need to distinguish between the diagonal case $\ell=\ell^{\prime}$ and the sums over $\ell>\ell^{\prime}$ and $\ell<\ell^{\prime}$. To do this, we introduce an even cut-off function $\eta_{0}$, identically 1 for $|\xi| \leq 1 / 4$ and identically 0 for $|\xi| \geq 1 / 2$, and the two cut-off 
functions $\eta_{ \pm}(\xi)=\chi_{(0,+\infty)}\left[1-\eta_{0}( \pm \xi)\right]$, supported respectively on $\ell>\ell^{\prime}$ and $\ell<\ell^{\prime}$. Accordingly, we decompose the sum above as

$$
K^{0}+K^{+}+K^{-}
$$

by writing $1=\eta_{0}\left(\ell^{\prime}-\ell\right)+\eta_{+}\left(\ell^{\prime}-\ell\right)+\eta_{-}\left(\ell^{\prime}-\ell\right)$.

The estimate for $K^{0}$ turns out to be trivial, while, in order to estimate $K^{ \pm}$, we need another decomposition. Clearly, it suffices to consider the case of $K^{+}$, which is supported when $\ell^{\prime}>\ell$.

We need to distinguish between the cases $\theta \leq 1 / \ell^{\prime}$ (recall that $\ell^{\prime}=\max \left\{\ell^{\prime}, \ell\right\}$ ) and $\theta>1 / \ell^{\prime}$. Thus we introduce a cut-off function $\chi_{1}$ supported in $[0,2]$, set $\chi_{2}=1-\chi_{2}$ and split $K^{+}$as $K_{1}^{+}(\omega, \theta)+K_{2}^{+}(\omega, \theta)$, where, for $j=1,2$,

$$
K_{j}^{+}(\omega, \theta):=\sum_{\ell, \ell^{\prime}=1}^{+\infty} e^{i t \lambda_{\ell, \ell^{\prime}}} \varphi\left(h^{2} \lambda_{\ell, \ell^{\prime}}\right) \psi\left(\ell^{\prime} / \ell\right) \eta_{+}\left(\ell^{\prime}-\ell\right) \chi_{j}\left(\left(\ell+\ell^{\prime}+n\right) \theta\right) Z_{\ell, \ell^{\prime}}(z, w) .
$$

Step 4. Here we prove the estimate for $K_{1}^{ \pm}(\omega, \theta)$.

Step 5. Finally, we prove the estimate for $K_{2}^{ \pm}(\omega, \theta)$ and complete the proof of Theorem 3.1

Step 1. We begin proving that the estimate (3.3) is trivial in two cases: when $h \geq \varepsilon_{0}$ and in the low frequency case, that is, when $|t| \leq h^{2}$. In this case, the oscillations of the exponential function are ineffective.

In $\mathrm{CaP} 1$ the authors proved a restriction-type lemma for blocks of spectral projections associated to the sublaplacian on $S^{2 n+1}$. A key ingredient in the proof was the following estimate.

Lemma 3.3. Let $1 \leq a<b$ be fixed. Then there exists a constant $C>0$ depending only on $n$ such that

$$
\sum_{\lambda_{\ell, \ell^{\prime}} \in(a, b]}\left(\ell+\ell^{\prime}\right) \leq C b(b-a+\log (b+1)) .
$$

Lemma 3.4. There exists a constant $C>0$ such that the estimates (3.3) and (3.4) hold in the following cases:

(i) when $t \in I_{2}$,

(ii) when $h \geq \varepsilon_{0}$, for all $t \in I_{s}$,

where $I_{2}$ and $I_{s}$ are defined in Theorem 3.1.

Proof. For $z, w \in S^{2 n+1},|t| \leq h^{2}$, using (2.7) and Lemma 3.3 we have

$$
\begin{aligned}
& \left|\sum_{a / h^{2}<\lambda_{\ell, \ell^{\prime}}<b / h^{2}} e^{i t \lambda_{\ell, \ell^{\prime}}} \varphi\left(h^{2} \lambda_{\ell, \ell^{\prime}}\right) Z_{\ell, \ell^{\prime}}(z, w)\right| \\
& \quad \leq \sum_{a / h^{2}<\lambda_{\ell, \ell^{\prime}}<b / h^{2}} \frac{d_{\ell, \ell^{\prime}}}{\omega_{2 n+1}} \leq \sum_{a / h^{2}<\lambda_{\ell, \ell^{\prime}}<b / h^{2}} \lambda_{\ell, \ell^{\prime}}^{n-1}\left(\ell+\ell^{\prime}\right) \\
& \quad \leq \frac{C}{h^{2 n-2}} \sum_{a / h^{2}<\lambda_{\ell, \ell^{\prime}}<b / h^{2}}\left(\ell+\ell^{\prime}\right) \leq \frac{C}{|h|^{2 n+2}},
\end{aligned}
$$

for a suitable positive constant $C$. 
If $|t| \leq h^{2}$, conclusion (i) follows at once. If $h \geq \varepsilon_{0}$, (ii) also follows at once, since

$$
h^{-(2 n+2)} \leq \varepsilon_{0}^{-2} h^{-2 n} \leq \varepsilon_{0}^{-2}|t|^{-Q / 2} .
$$

This proves the lemma.

Observe that this lemma in particular proves the estimate contained in (3.4) - in fact this is the trivial part of the estimate, and it provides no improvement with respect to the Sobolev embedding theorem.

Step 2. The dispersive estimate (3.1) also follows easily when $\langle z, w\rangle$ varies in a compact subset of the unit disk, as a consequence of the following result. First we recall the following estimates for Jacobi polynomials (see e.g. [BoCl, page 231]):

$$
\left|P_{\ell}^{(\alpha, \beta)}(\cos \theta)\right| \leq \begin{cases}C \ell^{\alpha} & \text { if } 0 \leq \theta \leq \frac{\pi}{2}, \\ C \ell^{-1 / 2} \theta^{-\alpha-\frac{1}{2}} & \text { if } 0<\theta \leq \frac{\pi}{2}, \\ C \ell^{-1 / 2}|\pi-\theta|^{-\beta-\frac{1}{2}} & \text { if } \frac{\pi}{2} \leq \theta<\pi, \\ C \ell^{\beta} & \text { if } \frac{\pi}{2} \leq \theta \leq \pi\end{cases}
$$

Lemma 3.5. Let $0<\varepsilon_{1}<\frac{\pi}{2}$ be fixed and set

$$
\mathcal{K}_{\varepsilon_{1}}:=\left\{(z, w) \in S^{2 n+1} \times S^{2 n+1}:\langle z, w\rangle=e^{i \omega} \cos \theta, \omega \in[0,2 \pi], \theta \in\left[\varepsilon_{1}, \pi / 2\right]\right\} .
$$

Then, there exists $C>0$ such that

$$
\sup _{(z, w) \in \mathcal{K}_{\varepsilon_{1}}}\left|\sum_{\ell, \ell^{\prime} \geq 0} e^{i t \lambda_{\ell, \ell^{\prime}}} \varphi\left(h^{2} \lambda_{\ell, \ell^{\prime}}\right) Z_{\ell, \ell^{\prime}}(z, w)\right| \leq \frac{C}{|t|^{Q / 2}} .
$$

Proof. The proof is simple since again in this case we do not need to consider the oscillations of the kernel. By symmetry in the parameters $\ell$ and $\ell^{\prime}$, in (3.7) it suffices to consider the case $\ell^{\prime} \geq \ell$.

Assume first that $\theta \in\left[\varepsilon_{1}, \frac{\pi}{4}\right]$. In this case, for $(z, w) \in \mathcal{K}_{\varepsilon_{1}}$, we have, using the first inequality in (3.6) and (2.2),

$$
\begin{aligned}
& \left|\sum_{\ell, \ell^{\prime} \geq 1} e^{i t \lambda_{\ell, \ell^{\prime}}} \varphi\left(h^{2} \lambda_{\ell, \ell^{\prime}}\right) Z_{\ell, \ell^{\prime}}(z, w)\right| \\
& \quad \leq C \sum_{\ell, \ell^{\prime} \geq 1} \frac{d_{\ell, \ell^{\prime}}}{\ell^{n-1}}(\cos \theta)^{\ell^{\prime}-\ell}\left|P_{\ell}^{\left(n-1, \ell^{\prime}-\ell\right)}(\cos 2 \theta)\right| \\
& \quad \leq \frac{C}{h^{2 n-2}} \sum_{a / h^{2} \leq \lambda_{\ell, \ell^{\prime}} \leq b / h^{2}}\left(\ell+\ell^{\prime}\right)(\cos \theta)^{\ell^{\prime}-\ell} \\
& \quad \leq \frac{C}{h^{2 n-2}}\left(\sum_{\ell=0}^{\lfloor c / h\rfloor} \sum_{\ell^{\prime}-\ell=0}^{\lfloor c / h\rfloor}\left(\ell^{\prime}-\ell\right)(\cos \theta)^{\ell^{\prime}-\ell}+\sum_{\ell=0}^{\lfloor c / h\rfloor} 2 \ell \sum_{\ell^{\prime}-\ell=0}^{\lfloor c / h\rfloor}(\cos \theta)^{\ell^{\prime}-\ell}\right) \leq \frac{C}{h^{2 n}},
\end{aligned}
$$

since $\theta \in\left[\varepsilon_{1}, \pi / 4\right]$.

Next, when $\theta \in[\pi / 4, \pi / 2)$, we observe that the sum vanishes when $\theta=\pi / 2$ and then we split it into two parts. Recalling that we are assuming $\ell \leq \ell^{\prime}$ we set

$$
\begin{aligned}
& E_{1}=\left\{\left(\ell, \ell^{\prime}\right) \in \mathbf{N}^{2}: a / h^{2}<\lambda_{\ell, \ell^{\prime}}<b / h^{2}, \ell>1 /|\pi-2 \theta|\right\}, \\
& E_{2}=\left\{\left(\ell, \ell^{\prime}\right) \in \mathbf{N}^{2}: a / h^{2}<\lambda_{\ell, \ell^{\prime}}<b / h^{2}, \ell \leq 1 /|\pi-2 \theta|\right\} .
\end{aligned}
$$


Then we have

$$
\begin{aligned}
& \left|\sum_{\ell^{\prime} \geq \ell \geq 1} e^{i t \lambda_{\ell, \ell^{\prime}}} \varphi\left(h^{2} \lambda_{\ell, \ell^{\prime}}\right) Z_{\ell, \ell^{\prime}}((z, w))\right| \\
& \quad \leq \sum_{\left(\ell, \ell^{\prime}\right) \in E_{1}} \varphi\left(h^{2} \lambda_{\ell, \ell^{\prime}}\right)\left|Z_{\ell, \ell^{\prime}}((z, w))\right|+\sum_{\left(\ell, \ell^{\prime}\right) \in E_{2}} \varphi\left(h^{2} \lambda_{\ell, \ell^{\prime}}\right)\left|Z_{\ell, \ell^{\prime}}((z, w))\right| \\
& \quad=: S_{1}+S_{2} .
\end{aligned}
$$

Using the last inequality in (3.6) we have

$$
\begin{aligned}
S_{1} & \leq C \sum_{\left(\ell, \ell^{\prime}\right) \in E_{1}} \frac{d_{\ell, \ell^{\prime}}}{\ell^{n-1}}(\cos \theta)^{\ell^{\prime}-\ell}\left|P_{\ell}^{\left(n-1, \ell^{\prime}-\ell\right)}(\cos 2 \theta)\right| \\
& \leq C \sum_{\left(\ell, \ell^{\prime}\right) \in E_{1}} \frac{d_{\ell, \ell^{\prime}}}{\ell^{n-1}}(\pi / 2-\theta)^{\ell^{\prime}-\ell} \ell^{\ell^{\prime}-\ell} \\
& \leq \frac{C}{h^{2 n-2}} \sum_{\ell=0}^{\lfloor c / h\rfloor} \sum_{\ell^{\prime}-\ell=0}^{\lfloor c / h\rfloor}\left(\frac{1}{\ell^{n-1}}\left(\ell^{\prime}-\ell\right) 2^{-\left(\ell^{\prime}-\ell\right)}+\frac{1}{\ell^{n-2}} 2^{-\left(\ell^{\prime}-\ell\right)}\right) \\
& \leq \frac{C}{h^{2 n}} .
\end{aligned}
$$

An analogous bound may be proved for $S_{2}$ using the third inequality in (3.6); we finally obtain (3.7).

\section{The main EStimate}

Step 3. Now we turn to the estimate (3.1). As a consequence of what has been proved in Steps 1-2, from now on we may assume that $h$ is sufficiently small, and precisely that $0<h<\varepsilon_{0}$, and that $t \in A$ where

$$
A:=\left\{t: h^{2} \leq|t| \leq c h^{s}\right\}
$$

Recall also that, because of the presence of the cut-off function $\psi$ in (3.1), we may consider the parameters $\ell, \ell^{\prime}$ to be such that $1 / M<\ell^{\prime} / \ell \leq M$, where $M>1$ is a fixed (large) constant.

Starting from (3.3) we now wish to show that for every $\kappa>0$ there exists $C>0$ such that

$$
\sup _{(z, w) \in \Omega}\left|\sum_{\ell, \ell^{\prime}=1}^{+\infty} e^{i t \lambda_{\ell, \ell^{\prime}}} \varphi\left(h^{2} \lambda_{\ell, \ell^{\prime}}\right) \psi\left(\ell^{\prime} \mid \ell\right) Z_{\ell, \ell^{\prime}}(z, w)\right| \leq C \frac{1}{h^{2 n+\kappa}}
$$

for all $t \in A$, where

$$
\Omega=\left\{(z, w) \in S^{2 n+1} \times S^{2 n+1}:\langle z, w\rangle e^{i \omega} \cos \theta \text {, where } 0 \leq \theta \leq \varepsilon_{1}, \omega \in[0,2 \pi)\right\} .
$$

We shall need to differentiate the proof between the cases $n=1$ and $n>1$ only at the end of Step 5, so that we will not distinguish between different values of $n$ until Proposition 4.11

We notice that we may assume $t>0$, since passing to the complex conjugate in (4.2) would change $Z_{\ell, \ell^{\prime}}$ into $Z_{\ell^{\prime}, \ell}$.

It turns out to be convenient to further simplify the problem by separating the cases $\ell=\ell^{\prime}, \ell<\ell^{\prime}$ and $\ell>\ell^{\prime}$.

We can do this by introducing yet another cut-off function. We let $\eta_{0}$ be an even cut-off function, identically 1 for $|\xi| \leq 1 / 4$ and identically 0 for $|\xi| \geq 1 / 2$. 
Then we write $1=\eta_{0}(\xi)+\eta_{-}(\xi)+\eta_{+}(\xi)$, where $\eta_{ \pm}(\xi)=\left(1-\eta_{0}(\xi)\right) \chi_{[0,+\infty)}( \pm \xi)$. Accordingly, we decompose the sum in (4.2) as

$$
K^{0}+K^{+}+K^{-} \text {. }
$$

It is easy to estimate $K^{0}$, since it coincides with the sum in (4.2) restricted to the diagonal terms $\ell=\ell^{\prime}$, and in this case the sum reduces to a summation in one variable.

Lemma 4.1. For all $t \in A$ we have

$$
\sup _{(z, w) \in \Omega}\left|\sum_{\ell, \ell^{\prime}=1}^{+\infty} e^{i t \lambda_{\ell, \ell^{\prime}}} \varphi\left(h^{2} \lambda_{\ell, \ell^{\prime}}\right) \eta_{0}\left(\ell-\ell^{\prime}\right) Z_{\ell, \ell^{\prime}}(z, w)\right| \leq C \frac{1}{h^{2 n}} .
$$

Proof. We easily check that

$$
\begin{gathered}
\sup _{(z, w) \in \Omega}\left|\sum_{\ell, \ell^{\prime}=1}^{+\infty} e^{i t \lambda_{\ell, \ell^{\prime}}} \varphi\left(h^{2} \lambda_{\ell, \ell^{\prime}}\right) \eta_{0}\left(\ell-\ell^{\prime}\right) Z_{\ell, \ell^{\prime}}(z, w)\right| \\
=\sup _{(z, w) \in \Omega}\left|\sum_{\ell=1}^{+\infty} e^{i t \lambda_{\ell, \ell}} \varphi\left(h^{2} \lambda_{\ell, \ell}\right) Z_{\ell, \ell}(z, w)\right| \\
\leq C\left|\sum_{\ell=1}^{+\infty} \varphi\left(h^{2} \lambda_{\ell, \ell}\right)\left(\lambda_{\ell, \ell}\right)^{n-1} \ell\right| \leq C \frac{1}{h^{2 n}}
\end{gathered}
$$

for all $t \in A$.

We are left with the estimate of $K^{ \pm}(\omega, \theta)$, where

$$
K^{ \pm}(\omega, \theta):=\sum_{\ell, \ell^{\prime}=0}^{\infty} e^{i t \lambda_{\ell, \ell^{\prime}}} \varphi\left(h^{2} \lambda_{\ell, \ell^{\prime}}\right) \eta_{ \pm}\left(\ell^{\prime}-\ell\right) \psi\left(\ell^{\prime} / \ell\right) Z_{\ell, \ell^{\prime}}(z, w)
$$

Our proof of (4.2), with the inner sum replaced by $K^{ \pm}$, hinges on Lemma 4.2 below and on Lemma 4.4, which will be proved in Step 4.

First, we need a representation of the Jacobi polynomials $P_{d}^{(\alpha, \beta)}$ showing explicitly the dependence on the parameters. To this end, we use a very precise representation of $P_{d}^{(\alpha, \beta)}$ due to A. Fitohui and M. M. Hamza [FiH]. We denote by $J_{\nu}$ the Bessel function of order $\nu$.

Lemma 4.2. Let $\alpha>-\frac{1}{2}, \beta>-1$, and $d$ be a positive integer. Then

$$
\begin{aligned}
& (\sin \theta)^{\alpha+1 / 2}(\cos \theta)^{\beta+1 / 2} P_{d}^{(\alpha, \beta)}(\cos 2 \theta) \\
& =\frac{\Gamma(d+\alpha+1)}{d !} \theta^{1 / 2}\left(\sum_{p=0}^{m} \theta^{p} Q_{2 p}(\beta, \theta) \frac{J_{\alpha+p}(N \theta)}{N^{\alpha+p}}+\theta^{m+1} \mathcal{R}_{m, N}(\theta)\right),
\end{aligned}
$$

where $N:=2 d+\alpha+\beta+1$, the functions $Q_{2 p}(\beta, \theta)$ are polynomials of degree $2 p$ in $\beta$ and analytic in $\theta \in[0, \pi / 2)$, and

$$
\mathcal{R}_{m, N}=\mathcal{O}\left(N^{-(\alpha+m+3 / 2)}\right),
$$

as $N \rightarrow+\infty$, uniformly in $\theta \in\left[0, \frac{\pi}{2}-\tilde{\varepsilon}\right]$, $\tilde{\varepsilon}>0$ being arbitrary. 
Proof. This is just a restatement of Theorem 4 in [FiH]; notice however that the parentheses are missing on the right-hand side of (6.6) in $[\mathrm{FiH}]$. By formula (6.6) in [FiH], setting $x=2 \theta$ and $B_{p}(x)=Q_{2 p}(\beta, \theta)$ (and calling $N$ what is called $2 N$ in [FiH]), we immediately obtain (4.6).

Next, the functions $B_{p}(x)$ are recursively defined by

$$
B_{0}(x)=1, \quad\left(x^{p+1} B_{p+1}(x)\right)^{\prime}=-\frac{1}{2} x^{p}\left(B_{p}^{\prime \prime}(x)+\frac{1-2 \alpha}{x} B_{p}^{\prime}(x)+\chi(x) B_{p}(x)\right),
$$

where

$$
\chi(x)=\left(\frac{1}{4}-\alpha^{2}\right)\left(\frac{1}{4 \sin ^{2}(x / 2)}-\frac{1}{x^{2}}\right)+\left(\frac{1}{4}-\beta^{2}\right) \frac{1}{4 \cos ^{2}(x / 2)},
$$

and it turns out that the $B_{p}(x)$ are analytic for $x \in[0, \pi)$ (see Section 6.3 in $[\mathrm{FiH}]$ ).

From the recursive relation (4.8) it is easy to see that the functions $B_{p}$ are polynomials of degree $2 p$ in the index $\beta$. (We point out that, for our purposes, the dependence on $\alpha$ is not relevant, since $\alpha=n-1$, and it is not related to the indexes $\ell, \ell^{\prime}$, while $\beta=\left|\ell^{\prime}-\ell\right|$.) In fact, the recursive relation can be restated by saying that

$$
-2 B_{p+1}=H_{p+1}\left((L+\chi) B_{p}\right),
$$

where $H_{p+1}$ is the integral operator (3.3) in [FiH], independent of $\alpha$ and $\beta$, and $L$ is the differential operator given by $L u=u^{\prime \prime}+\frac{1-2 \alpha}{x} u^{\prime}$. Now, the statement about the dependence on $\beta$ follows easily by induction. Hence, $Q_{2 p}(\beta, \theta)$ is a polynomial of degree $2 p$ in $\beta$ and analytic in $\theta \in\left[0, \frac{\pi}{2}\right)$.

The statement about the remainder term $\mathcal{R}_{m, N}(\theta)$ is explicit in Theorem 4 (see formula (6.6) again) in $[\mathrm{FiH}]$.

We are going to apply Lemma 4.2 so that we observe that in our case $\alpha=n-1$, $d=\min \left\{\ell, \ell^{\prime}\right\}$ and $\beta=\left|\ell^{\prime}-\ell\right|$; hence $N=\ell+\ell^{\prime}+n$.

In what follows we denote by $g_{j}, \tilde{g}_{j^{\prime}}$ polynomials of degree $j, j^{\prime}$ resp., in the indicated variables, that again may have a different expression from one line to the next. Then, we write

$$
\frac{\ell+\ell^{\prime}+n}{\ell^{\prime}}\left(\begin{array}{c}
\ell^{\prime}+n-1 \\
\ell^{\prime}-1
\end{array}\right)=\left(\ell+\ell^{\prime}+n\right) \frac{\left(\ell^{\prime}+n-1\right) !}{\ell^{\prime} ! n !}=N g_{n-1}\left(\ell^{\prime}\right)
$$

and

$$
\frac{\Gamma(\ell+n)}{\ell !}=\tilde{g}_{n-1}(\ell) .
$$

Then, using (2.6), (4.9) and (4.10), writing $\langle z, w\rangle=e^{i \omega} \cos \theta$, in the case $\ell^{\prime} \geq \ell$ we have

$$
\begin{aligned}
Z_{\ell, \ell^{\prime}}(z, w)= & \frac{n}{\omega_{2 n+1}} N g_{n-1}\left(\ell^{\prime}\right) e^{i \omega\left(\ell^{\prime}-\ell\right)}(\sin \theta)^{-n+1 / 2}(\cos \theta)^{-1 / 2} \tilde{g}_{n-1}(\ell) \theta^{1 / 2} \\
\times & \left(\sum_{p=0}^{m} \theta^{p} Q_{2 p}(\beta, \theta) \frac{J_{n-1+p}(N \theta)}{N^{n-1+p}}+\theta^{m+1} \mathcal{R}_{m, N}(\theta)\right) \\
=b(\theta) e^{i \omega\left(\ell^{\prime}-\ell\right)} & N g_{n-1}\left(\ell^{\prime}\right) \tilde{g}_{n-1}(\ell) \\
& \times\left(\sum_{p=0}^{m} \theta^{2 p} Q_{2 p}(\beta, \theta) \frac{J_{n-1+p}(N \theta)}{(N \theta)^{n-1+p}}+\theta^{m-n+2} \mathcal{R}_{m, N}(\theta)\right),
\end{aligned}
$$

where $b$ denotes an entire function of $\theta$. 
If $\ell \geq \ell^{\prime}$ we simply switch the roles between $\ell$ and $\ell^{\prime}$ in the formula above.

Then, in order to estimate (4.2) it suffices to bound the modulus of

$$
\begin{aligned}
e^{i\left[t \lambda_{\ell, \ell^{\prime}}+\omega\left(\ell^{\prime}-\ell\right)\right]} & \varphi\left(h^{2} \lambda_{\ell, \ell^{\prime}}\right) \psi\left(\ell^{\prime} / \ell\right) \eta_{ \pm}\left(\ell-\ell^{\prime}\right) N g_{n-1}\left(\ell^{\prime}\right) \tilde{g}_{n-1}(\ell) \\
& \times\left(\sum_{p=0}^{m} \theta^{2 p} Q_{2 p}(\beta, \theta) \frac{J_{n-1+p}(N \theta)}{(N \theta)^{n-1+p}}+\theta^{m-n+2} \mathcal{R}_{m, N}(\theta)\right) .
\end{aligned}
$$

We need to distinguish the cases when $N \theta$ remains bounded and when it is bounded from below. Then, let $\chi_{1}$ be a smooth cut-off function with compact support such that $0 \leq \chi_{1} \leq 1, \chi_{1}(x)=1$ for $0 \leq x \leq 1$ and $\chi_{1}(x)=0$ for $x \geq 2$, and set $\chi_{2}=1-\chi_{1}$. Therefore, for $j=1,2$ we write

$$
\begin{aligned}
K_{j}^{ \pm}(\omega, \theta):= & \sum_{a / h^{2}<\lambda_{\ell, \ell^{\prime}}<b / h^{2}} e^{i\left[t \lambda_{\ell, \ell^{\prime}}+\omega\left(\ell^{\prime}-\ell\right)\right]} \varphi\left(h^{2} \lambda_{\ell, \ell^{\prime}}\right) \psi\left(\ell^{\prime} / \ell\right) \\
\times & \eta_{ \pm}\left(\ell-\ell^{\prime}\right) N g_{n-1}\left(\ell^{\prime}\right) \tilde{g}_{n-1}(\ell) \chi_{j}(N \theta) \\
& \times\left(\sum_{p=0}^{m} \theta^{2 p} Q_{2 p}(\beta, \theta) \frac{J_{n-1+p}(N \theta)}{(N \theta)^{n-1+p}}+\theta^{m-n+2} \mathcal{R}_{m, N}(\theta)\right) .
\end{aligned}
$$

Remark 4.3. We observe that the eigenvalue $\lambda_{\ell, \ell^{\prime}}=2\left[(\ell+n / 2)\left(\ell^{\prime}+n / 2\right)-n^{2} / 4\right]$ and we set

$$
k=\ell+\frac{n}{2}, \quad k^{\prime}=\ell^{\prime}+\frac{n}{2} .
$$

Notice that we may consider the quantities $g_{n-1}, \tilde{g}_{n-1}$ as functions of $k, k^{\prime}$ resp., and write $N=k+k^{\prime}$. We adopt the convention that if $n$ is odd, then the symbol $\sum_{k, k^{\prime} \geq 1}$ shall denote the sum over a suitable subset of $\mathbf{N}$ shifted by $1 / 2$.

Moreover, the condition $h^{2} \lambda_{\ell, \ell^{\prime}} \in \operatorname{supp} \varphi$ becomes

$$
\frac{a_{h}}{h^{2}} \leq k k^{\prime} \leq \frac{b_{h}}{h^{2}}
$$

where we set

$$
a_{h}=\frac{a}{2}+\frac{h^{2} n^{2}}{4} \quad \text { and } \quad b_{h}=\frac{b}{2}+\frac{h^{2} n^{2}}{4} .
$$

For simplicity of notation we take $0<a^{\prime} \leq a_{h}$ and $b^{\prime} \geq b_{h}$ for all $h \leq 1$. We also set $c^{\prime}=\sqrt{b^{\prime}}$.

The cut-off function $\varphi\left(h^{2} \lambda_{\ell, \ell^{\prime}}\right)$ can be written as

$$
\varphi\left(h^{2} \lambda_{\ell, \ell^{\prime}}\right)=\varphi\left(h^{2}\left(2 k k^{\prime}-n^{2} / 2\right)\right)=: \varphi_{h}\left(h^{2} k k^{\prime}\right) .
$$

We remark that Lemma 4.4 holds true if the cut-off function $\varphi$ is replaced by a family of functions $\varphi_{\varepsilon}$ converging in the Schwartz norms to $\varphi$ as $\varepsilon \rightarrow 0$. Since the dependence on $h$ of $\varphi_{h}$ is ineffective, with an abuse of notation, we again write $\varphi$ to denote the functions $\varphi_{h}$.

The cut-off function $\psi\left(\ell / \ell^{\prime}\right)$, supported when $1 / M \leq \ell / \ell^{\prime} \leq M$, is changed into

$$
\psi\left((k-n / 2) /\left(k^{\prime}-n / 2\right)\right)=: \tilde{\psi}\left(k, k^{\prime}\right) .
$$

Observed that $\tilde{\psi}$ is a cut-off function having support contained in the set $\{1 \leq$ $\left.k / k^{\prime} \leq M\right\}$. Finally, notice that the support condition of $\varphi$ implies that

$$
n / 2 \leq k, k^{\prime} \leq c^{\prime} / h .
$$


With the change of parameters (4.13), the quantity $\beta$ remains unchanged, and the phase function $t\left[\lambda_{\ell, \ell^{\prime}}+\omega \beta\right]$ becomes $2 t\left[k k^{\prime}+\omega \beta-n^{2} / 4\right]$ and we may absorb the factor 2 in the parameter $t$.

Step 4. We now wish to estimate the modulus of $K_{1}^{ \pm}(\omega, \theta)$, as defined in (4.12).

We will consider the case of $K_{1}^{+}$, the other one being completely analogous.

It suffices to estimate the modulus of

$$
\begin{array}{r}
\sum_{p=0}^{m}\left(\sum_{k, k^{\prime} \geq n / 2} e^{i\left[t k k^{\prime}+\omega\left(k^{\prime}-k\right)\right]} \varphi\left(h^{2} k k^{\prime}\right) \tilde{\psi}\left(k, k^{\prime}\right) \chi_{1}(N \theta) \eta_{+}\left(k^{\prime}-k\right) N g_{n-1}\left(k^{\prime}\right) \tilde{g}_{n-1}(k)\right. \\
\left.\times \theta^{2 p} Q_{2 p}(\beta, \theta) \frac{J_{n-1+p}(N \theta)}{(N \theta)^{n-1+p}}\right) \\
+\sum_{k, k^{\prime} \geq n / 2} e^{i\left[t k k^{\prime}+\omega\left(k^{\prime}-k\right)\right]} \varphi\left(h^{2} k k^{\prime}\right) \tilde{\psi}\left(k, k^{\prime}\right) \eta_{+}\left(k^{\prime}-k\right) \chi_{1}(N \theta) \\
\times N g_{n-1}\left(k^{\prime}\right) \tilde{g}_{n-1}(k) \theta^{m-n+2} \mathcal{R}_{m, N}(\theta)
\end{array}
$$

$$
=: \sum_{p=0}^{m} K_{p}^{1,+}(\omega, \theta)+K_{\mathcal{R}}^{1,+}(\omega, \theta) .
$$

Since $\mathcal{R}_{m, N}(\theta)=\mathcal{O}\left(\frac{1}{N^{n+m+1 / 2}}\right)$ uniformly for $\theta \in\left[0, \frac{\pi}{2}-\varepsilon_{1}\right]$, for $\varepsilon_{1}>0$, we can easily estimate the modulus of the error term $K_{\mathcal{R}}^{1,+}(\omega, \theta, N)$ by simply taking the modulus inside the sum. Observing that $x \mapsto x^{n-1} \varphi(x)$ is also a smooth function with compact support, and choosing $m=\max (n-2,0)$,

$$
\begin{aligned}
\left|K_{\mathcal{R}}^{1,+}(\omega, \theta)\right|=\mid \sum_{k, k^{\prime} \geq n / 2} e^{i\left[t k k^{\prime}+\omega\left(k^{\prime}-k\right)\right]} \varphi\left(h^{2} k k^{\prime}\right) \tilde{\psi}\left(k, k^{\prime}\right) \eta_{+}\left(k^{\prime}-k\right) \chi_{1}(N \theta) \\
\quad \times N g_{n-1}\left(k^{\prime}\right) \tilde{g}_{n-1}(k) \theta^{m-n+2} \mathcal{R}_{m, N}(\theta) \mid \\
\leq \frac{C}{h^{2 n-2}} \sum_{k=n / 2}^{\left\lfloor c^{\prime} / h\right\rfloor} \sum_{k^{\prime}=n / 2}^{\left\lfloor c^{\prime} / h\right\rfloor}\left|\varphi\left(h^{2} k k^{\prime}\right)\left(h^{2} k k^{\prime}\right)^{n-1} \frac{1}{N^{n+m-1 / 2}}\right| \\
\leq \frac{C}{h^{2 n-2}} \sum_{k=n / 2}^{\left\lfloor c^{\prime} / h\right\rfloor} \sum_{k^{\prime}=n / 2}^{\left\lfloor c^{\prime} / h\right\rfloor} \frac{1}{\left(k+k^{\prime}\right)^{1 / 2}} \\
\leq \frac{C}{h^{2 n-1 / 2}},
\end{aligned}
$$

uniformly in $\omega$ and $\theta \in\left[0, \varepsilon_{1}\right]$. Therefore, with $m=\max (n-2,0)$ we have

$$
\left|K_{\mathcal{R}}^{1,+}(\omega, \theta)\right| \leq \frac{C}{h^{2 n-1 / 2}}
$$

for all $n \geq 1$.

We turn to the estimate of the main term in (4.14), with $m=\max (n-2,0)$.

In order to take advantage of the oscillations of the kernel we need the following estimate for oscillating sums. We denote by $\hat{f}(\xi)$ the Fourier transform of an integrable function $f$ and defined by $\hat{f}(\xi)=\int_{\mathbf{R}} f(x) e^{-2 \pi i x \xi} d x$. 
Lemma 4.4. Let $\varphi \in \mathcal{C}_{0}^{\infty}(\mathbf{R}), \operatorname{supp} \varphi \subseteq[a, b], 0<a<b<\infty$, and let $\sigma$ be $a$ symbol in $\mathcal{S}^{0}$. Let $\mu \in \mathbf{R}$ and set $\operatorname{dist}(\mu, \mathbf{Z}) \geq \delta$, for some $\delta>0$. Then for every $L>1$ there exists a positive constant $C_{L}>0$, depending only on $\varphi$ and $\sigma$, such that for $\delta, \varepsilon>0$ with $0<\varepsilon \leq \delta$, we have that

$$
\left|\sum_{k \in \mathbf{Z}} e^{2 \pi i \mu k} \varphi(\varepsilon k) \sigma(k)\right| \leq C_{L} \max \left\{\frac{\varepsilon^{L-1}}{\delta^{L}}, 1\right\},
$$

as $\varepsilon \rightarrow 0$.

Proof. Let $\psi, \tau$ be such that $\varphi=\widehat{\psi}, \sigma=\widehat{\tau}$.

We consider first the case $\sigma=1$. By the classical Poisson summation formula

$$
\begin{aligned}
\left|\sum_{k \in \mathbf{Z}} e^{2 \pi i \mu k} \varphi(\varepsilon k)\right| & =\left|\sum_{k \in \mathbf{Z}} e^{2 \pi i \mu k} \widehat{\psi_{\varepsilon}}(k)\right|=\left|\sum_{k^{\prime} \in \mathbf{Z}} \psi_{\varepsilon}\left(k^{\prime}+\mu\right)\right| \\
& \leq \frac{C_{L}}{\varepsilon} \sum_{k^{\prime} \in \mathbf{Z}} \frac{1}{\left(1+\frac{\left|k^{\prime}+\mu\right|}{\varepsilon}\right)^{L}},
\end{aligned}
$$

where $\psi_{\varepsilon}(x)=\varepsilon^{-1} \psi\left(\varepsilon^{-1} x\right)$.

We may assume $|\mu| \leq \frac{1}{2}$, so that $\operatorname{dist}(\mu, \mathbf{Z})=|\mu| \geq \delta$ and

$$
\begin{aligned}
\sum_{k^{\prime} \in \mathbf{Z}} \frac{1}{\left(1+\frac{\left|k^{\prime}+\mu\right|}{\varepsilon}\right)^{L}} & \leq \frac{1}{\left(1+\frac{|\mu|}{\varepsilon}\right)^{L}}+2 \int_{-\infty}^{+\infty} \frac{1}{\left(1+\frac{|x+\mu|}{\varepsilon}\right)^{L}} d x \\
& \leq \frac{1}{\left(1+\frac{\delta}{\varepsilon}\right)^{L}}+2 \varepsilon \int_{-\infty}^{+\infty} \frac{1}{(1+|y|)^{L}} d y
\end{aligned}
$$

Therefore,

$$
\begin{aligned}
\left|\sum_{k \in \mathbf{Z}} e^{2 \pi i \mu k} \varphi(\varepsilon k)\right| & \leq \frac{C_{L}}{\varepsilon}\left(\frac{1}{\left(1+\frac{\delta}{\varepsilon}\right)^{L}}+2 \varepsilon \int_{-\infty}^{+\infty} \frac{1}{(1+|y|)^{L}} d y\right) \\
& \leq C_{L} \frac{\varepsilon^{L-1}}{(\varepsilon+\delta)^{L}}+C \\
& \leq C_{L} \max \left\{\frac{\varepsilon^{L-1}}{\delta^{L}}, 1\right\},
\end{aligned}
$$

proving (4.17) in the case $\sigma=1$.

Next we suppose that $\sigma$ belongs to classical symbol class $\mathcal{S}^{0}$. Notice that

$$
\varphi(\varepsilon k) \sigma(k)=\widehat{\psi_{\varepsilon}}(k) \widehat{\tau}(k)=\left(\psi_{\varepsilon} * \tau\right) \widehat{(}(k)=\left(\left(\psi * \tau_{1 / \varepsilon}\right)_{\varepsilon}\right) \hat{(}(k) .
$$

Thus we may repeat the previous arguments as in (4.18) to obtain

$$
\begin{aligned}
\left|\sum_{k \in \mathbf{Z}} e^{2 \pi i \mu k} \varphi(\varepsilon k) \sigma(k)\right| & =\left|\sum_{k \in \mathbf{Z}} e^{2 \pi i \mu k}\left(\left(\psi * \tau_{1 / \varepsilon}\right)_{\varepsilon}\right) \hat{(}(k)\right| \\
& =\left|\sum_{k^{\prime} \in \mathbf{Z}}\left(\psi * \tau_{1 / \varepsilon}\right)_{\varepsilon}\left(k^{\prime}+\mu\right)\right| \\
& \leq \frac{C_{L}}{\varepsilon} \sum_{k^{\prime} \in \mathbf{Z}} \frac{1}{\left(1+\frac{\left|k^{\prime}+\mu\right|}{\varepsilon}\right)^{L}},
\end{aligned}
$$

where $C_{L}$ does not depend on $\varepsilon$ as long as the Schwartz norms of $\left(\psi * \tau_{1 / \varepsilon}\right)$ are uniformly bounded in $\varepsilon$. This happens if and only if the Schwartz norms of $\left(\psi * \tau_{1 / \varepsilon}\right)$ 
are uniformly bounded in $\varepsilon$, as $\varepsilon \rightarrow 0$. Now

$$
\left(\psi * \tau_{1 / \varepsilon}\right) \widehat{)}(x)=\widehat{\psi}(x) \widehat{\tau_{1 / \varepsilon}}(x)=\varphi(x) \sigma(x / \varepsilon) .
$$

Since $\sigma \in \mathcal{S}^{0}$, it is straightforward to check that

$$
\begin{aligned}
\left|D_{x}^{j}(\varphi \sigma(\cdot / \varepsilon))(x)\right| & =\left|\sum_{j^{\prime}=0}^{j} c_{j^{\prime}} \varphi^{\left(j-j^{\prime}\right)}(x) \frac{1}{\varepsilon^{j^{\prime}}} \sigma^{\left(j^{\prime}\right)}(x / \varepsilon)\right| \\
& \leq C \sum_{j^{\prime}=0}^{j}\left|\varphi^{\left(j-j^{\prime}\right)}(x)\right| \frac{1}{\varepsilon^{j^{\prime}}} \frac{1}{(1+|x| / \varepsilon)^{j^{\prime}}} \\
& \leq C \sum_{j^{\prime}=0}^{j}\left|\varphi^{\left(j-j^{\prime}\right)}(x)\right| \frac{1}{\left(\varepsilon^{j^{\prime}}+a\right)^{j^{\prime}}},
\end{aligned}
$$

since $\operatorname{supp} \varphi \subset[a, b]$ and $a>0$. The statement now follows.

Remark 4.5. It is worth noticing that, by choosing as a symbol $\sigma$ a smooth cutoff function with compact support, the estimate (4.17) may be proved also for truncated sums.

Lemma 4.6. For $N=k^{\prime}+k$ and $\beta=\left|k^{\prime}-k\right|$ set

$$
\sigma_{1}\left(k, k^{\prime}\right)=\eta_{+}\left(k^{\prime}-k\right) \tilde{\psi}\left(k, k^{\prime}\right) \theta^{2 p} Q_{2 p}(\beta, \theta) \frac{J_{n-1+p}(N \theta)}{(N \theta)^{n-1+p}} \chi_{1}(N \theta) .
$$

Then $\sigma_{1}$ is a symbol of order 0 in $k^{\prime}$, depending on the parameters $\theta$ and $k$, with norm uniformly bounded in such parameters.

Proof. We wish to show that, considering $k^{\prime}=\xi$ as a continuous parameter, $\sigma_{1}$ is a smooth function of $\xi$ and, for each non-negative integer $k$, there exists a positive constant $C=C_{k}$, independent of $k$ and $\theta \in\left[0, \pi / 2-\varepsilon_{1}\right]$, such that

$$
\left|\partial_{\xi}^{k} \sigma_{1}(\xi)\right| \leq C(1+|\xi|)^{-k} .
$$

Notice that since $k^{\prime} \geq 1$ we may assume that we have extended $\sigma_{1}$ to be identically 0 when $\xi \leq 1 / 2$.

Since the Bessel function $J_{\nu}$ of integral order $\nu$ is analytic and has a zero of order $\nu$ at the origin, it is clear that $\sigma_{1}$ is smooth and bounded uniformly in $\theta$. Moreover, recall from Lemma 4.2 that $Q_{2 p}(\beta, \theta)$ is a polynomial of degree $2 p$ in $\beta$ and analytic in $\theta \in\left[0, \frac{\pi}{2}\right)$. Hence, since $\chi_{1}(N \theta)=0$ for $N \theta \geq 2$, we have that, on the support of $\chi_{1}, \theta \leq 2 / N \leq C / \xi$, implying that

$$
\left|\theta^{2 p} Q_{2 p}(\beta, \theta)\right| \leq C \frac{\left|Q_{2 p}(\beta, \theta)\right|}{N^{2 p}},
$$

which is bounded, as $\xi \rightarrow+\infty$, uniformly in $\theta$.

Next we consider the derivatives. If the derivative falls on the factor $\theta^{2 p} Q_{2 p}(\beta, \theta)$ we simply lower the degree of the polynomial of $\xi$ and then obtain the estimate

$$
\left|\partial_{\xi}\left[\theta^{2 p} Q_{2 p}(\beta, \theta)\right]\right| \leq C \frac{1}{\xi},
$$

as $\xi \rightarrow+\infty$, uniformly in $\theta$, as we required. 
It the derivative falls on the factor $\frac{J_{n-1+p}(N \theta)}{(N \theta)^{n-1+p}}$, since the derivative of the Bessel function of order $\nu$ satisfies the identity $J_{\nu}^{\prime}(z)-\frac{\nu}{z} J_{\nu}(z)+J_{\nu-1}(z)$, again we obtain that

$$
\left|\partial_{\xi}\left[\frac{J_{n-1+p}(N \theta)}{(N \theta)^{n-1+p}}\right]\right| \leq C\left(\theta+\frac{1}{N}\right) \leq C \frac{1}{\xi},
$$

as $\xi \rightarrow+\infty$, uniformly in $\theta$.

If the derivative falls on $\chi_{1}$ it produces an extra factor $\theta$, which is less than $C / \xi$.

Hence,

$$
\left|\partial_{\xi} \sigma_{1}(\xi)\right| \leq C(1+|\xi|)^{-1}
$$

as $|\xi| \rightarrow+\infty$, uniformly in $\theta$.

Finally, if the derivative falls on $\tilde{\psi}$ it produces a factor of the order of $k / \xi^{2}$ which is less than or equal to $C /|\xi|$, as $\xi \rightarrow+\infty$.

The argument can be repeated for all higher order derivatives, so the lemma is proven.

We wish to apply Lemma 4.4, and this leads us to analize the phase function $t k k^{\prime}+\omega\left(k^{\prime}-k\right)$. Recall that $A$ has been defined in (4.1) and that, as observed earlier, by passing to the complex conjugate, we may assume $t>0$. We then introduce the set of indices in $\mathbf{N}^{2}$ :

$\mathcal{V}=\left\{\left(k, k^{\prime}\right): k, k^{\prime} \geq n / 2, a^{\prime} / h^{2} \leq k k^{\prime} \leq b^{\prime} / h^{2}, 1 / M \leq k / k^{\prime} \leq M,\left|k-k^{\prime}\right| \geq 1 / 2\right\}$.

We set moreover

$$
\mathcal{V}_{+}=\mathcal{V} \cap\left\{\left(k, k^{\prime}\right): k^{\prime}>k\right\} \quad \text { and } \quad \mathcal{V}_{-}=\mathcal{V} \cap\left\{\left(k, k^{\prime}\right): k^{\prime}<k\right\}
$$

Finally, we introduce the space of parameters $(t, \omega)$

$$
R:=\left[h^{2}, h^{s}\right] \times[0,2 \pi) \subset A \times[0,2 \pi) .
$$

Lemma 4.7. On $\mathcal{V}_{+}$we set $\mu(k):=(t k+\omega) / 2 \pi$ and $\mu^{\prime}\left(k^{\prime}\right)=\left(\omega-t k^{\prime}\right) / 2 \pi$. Then there exist a constant $0<\gamma<1$ and two regions $R_{I}, R_{I I}$ in the $(t, \omega)$-space such that $R \subseteq \cup R_{I}$ and for all $(t, \omega) \in R_{i}, i \in\{I, I I\}$, one between the following two conditions:

(I) $\operatorname{dist}(\mu(k), \mathbf{Z}) \geq \gamma$ tk for all $\left(k, k^{\prime}\right) \in \mathcal{V}_{+}$;

(II) $\operatorname{dist}\left(\mu^{\prime}\left(k^{\prime}\right), \mathbf{Z}\right) \geq \gamma t k^{\prime}$ for all $\left(k, k^{\prime}\right) \in \mathcal{V}_{+}$

holds.

An analogous statement holds in the case of $\mathcal{V}_{-}$.

Proof. We begin by observing that $\left(k, k^{\prime}\right) \in \mathcal{V}_{+}$implies that $1 \leq k<k^{\prime} \leq\left\lfloor c^{\prime} / h\right\rfloor$ so that, for $t \in\left[h^{2}, h^{s}\right]$, we have

$$
\frac{h^{2}}{2 \pi} \leq \frac{1}{2 \pi} t k \leq \frac{1}{2 \pi} t k^{\prime} \leq c h^{s-1} \leq c_{1} /(4 \pi),
$$

if $h \leq \varepsilon_{0}$ is sufficiently small and for some positive, small enough $c_{1}$.

Let $R_{I}=\left\{(t, \omega) \in\left[h^{2}, h^{s}\right] \times[0,2 \pi): 0<c_{1}<\omega<2 \pi-c_{1}\right\}$, with $c_{1}$ as above. Then (I) holds for $(t, \omega) \in R_{I}$ since for $m$ an integer,

$$
\left|\frac{t k+\omega}{2 \pi}-m\right| \geq\left|\frac{\omega}{2 \pi}-m\right|-\frac{c_{1}}{4 \pi} \geq \frac{c_{1}}{4 \pi} .
$$

Replacing $\omega$ by $2 \pi-\omega$ we may assume now that $-c_{1}<\omega<c_{1}$. In this case, notice that $\operatorname{dist}(\mu, \mathbf{Z})=\frac{1}{2 \pi}|t k+\omega|$ and $\operatorname{dist}\left(\mu^{\prime}, \mathbf{Z}\right)=\frac{1}{2 \pi}\left|t k^{\prime}-\omega\right|$. 
Then, if $\omega>0$, we have that $\frac{t k+\omega}{t k} \geq 1$, so that in this case (I) holds.

If $\omega<0$, we then have that $\frac{t k^{\prime}-\omega}{t k^{\prime}} \geq 1$, so that in this case (II) holds.

Proposition 4.8. There exists a constant $C>0$ such that

$$
\left|K_{p}^{1,+}(\omega, \theta)\right| \leq C \frac{1}{|t|^{n+1}}
$$

uniformly in $\omega$ and $\theta \in\left[0, \varepsilon_{1}\right]$, for all $|t| \in\left[h^{2}, c h^{s}\right]$.

Proof. Recall the definition of $K_{p}^{1, \pm}(\omega, \theta)$ introduced in (4.14).

Using Lemma 4.6 we have that

$$
K_{p}^{1,+}(\omega, \theta)=\sum_{k, k^{\prime} \geq 1} e^{i\left[t k k^{\prime}+\omega\left|k^{\prime}-k\right|\right]} \varphi\left(h^{2} k k^{\prime}\right) N g_{n-1}\left(k^{\prime}\right) \tilde{g}_{n-1}(k) \sigma_{1}\left(k^{\prime}\right) .
$$

Notice that $g_{n-1}\left(k^{\prime}\right) \tilde{g}_{n-1}(k)=k^{n-1} k^{\prime n-1}+$ lower order terms. Thus, we estimate the higher order terms, the other ones being estimated in a similar way, giving rise to a better bound. Hence, it suffices to estimate

$$
\left|\sum_{k, k^{\prime} \geq n / 2} e^{i\left[t k k^{\prime}+\omega\left(k^{\prime}-k\right)\right]} \varphi\left(h^{2} k k^{\prime}\right) N k^{n-1} k^{\prime n-1} \sigma_{1}\left(k^{\prime}\right)\right|,
$$

and we distiguish two cases according to whether condition (I) or (II) in Lemma 4.7 holds, respectively.

Case (I). We assume that $\operatorname{dist}(\mu(k), \mathbf{Z}) \geq \gamma t k$ for all $\left(k, k^{\prime}\right) \in \mathcal{V}_{+}$and for some $0<\gamma<1$, so that $|t k+\omega| \geq 2 \pi \gamma t k$ for all $k \geq 1$. Starting from (4.22), we wish to estimate

$$
\begin{aligned}
& \left|\sum_{k=n / 2}^{\left\lfloor c^{\prime} / h\right\rfloor} \sum_{k^{\prime}=n / 2}^{\left\lfloor c^{\prime} / h\right\rfloor} e^{i\left[t k k^{\prime}+\omega\left(k^{\prime}-k\right)\right]} \varphi\left(h^{2} k k^{\prime}\right) N k^{n-1} k^{\prime n-1} \sigma_{1}\left(k, k^{\prime}\right)\right| \\
& \leq \frac{C}{h^{2 n}} \sum_{k=n / 2}^{\left\lfloor c^{\prime} / h\right\rfloor} \frac{1}{k}\left|\sum_{k^{\prime}=n / 2}^{\left\lfloor c^{\prime} / h\right\rfloor} e^{i k^{\prime}[t k+\omega]} \varphi\left(h^{2} k k^{\prime}\right)\left(h^{2} k k^{\prime}\right)^{n} \sigma_{1}\left(k, k^{\prime}\right)\right| \\
& \quad+\frac{C}{h^{2 n-2}} \sum_{k=n / 2}^{\left\lfloor c^{\prime} / h\right\rfloor} k\left|\sum_{k^{\prime}=n / 2}^{\left\lfloor c^{\prime} / h\right\rfloor} e^{i k^{\prime}[t k+\omega]} \varphi\left(h^{2} k k^{\prime}\right)\left(h^{2} k k^{\prime}\right)^{n-1} \sigma_{1}\left(k, k^{\prime}\right)\right| .
\end{aligned}
$$

Notice that it suffices to bound the first sum on the right-hand side of (4.23) above.

We now apply Lemma 4.4 to the inner sum of the first term on the right-hand side of (4.23) above, with

$$
\mu=[t k+\omega] / 2 \pi, \quad \delta=\gamma t k \geq \gamma h^{2} k=\varepsilon,
$$

and cut-off function $x \mapsto x^{n} \varphi$ and with the aid of Lemma 4.6. 
Hence we obtain that for every $L>1$ the right-hand side of (4.23) is less than or equal to a constant times

$$
\begin{aligned}
\frac{C_{L}}{h^{2 n}} \sum_{k=n / 2}^{\left\lfloor c^{\prime} / h\right\rfloor} \frac{1}{k} \max \left(\frac{\varepsilon^{L-1}}{\delta^{L}}, 1\right) & \leq \frac{C}{h^{2 n}} \sum_{k=n / 2}^{\left\lfloor c^{\prime} / h\right\rfloor} \frac{1}{k}\left(\frac{\left(h^{2} k\right)^{(L-1)}}{(t k)^{L}}+1\right) \\
& \leq \frac{C}{h^{2 n}}\left(\frac{h^{2(L-1)}}{t^{L}} \sum_{k=n / 2}^{\left\lfloor c^{\prime} / h\right\rfloor} \frac{1}{k^{2}}+\log (1 / h)\right) \\
& \leq C\left(\frac{1}{t^{n+1}}+\frac{1}{h^{2(n+\kappa)}}\right)
\end{aligned}
$$

for any given $\kappa>0$, if we choose $L=n+1$.

Therefore, for $t$ such that $h^{2} \leq t \leq h^{s}$ for every $s>s_{n}$, we have

$$
\frac{1}{h^{2(n+\kappa)}} \leq \frac{C}{t^{2(n+\kappa) / s}} \leq \frac{C}{t^{n+1}}
$$

Hence, for all $t \in A$ we obtain

$$
\left|K_{p}^{1,+}(\omega, \theta)\right| \leq C \frac{1}{|t|^{n+1}}
$$

This proves the statement in Case (I).

Case (II). We now assume that $\operatorname{dist}\left(\mu^{\prime}\left(k^{\prime}\right), \mathbf{Z}\right) \geq \gamma t k^{\prime}$ for all $\left(k, k^{\prime}\right) \in \mathcal{V}_{+}$and for some $0<\gamma^{\prime}<1$, so that $\left|t k^{\prime}-\omega\right| \geq 2 \pi\left(h^{2} k^{\prime}\right)^{\gamma^{\prime}}$, all $k^{\prime} \in \mathbf{N}$. In this case, again we start from (4.22), apply Lemma 4.4 to the inner sum with

$$
\mu=\left[t k^{\prime}-\omega\right] / 2 \pi, \quad \delta=\gamma t k^{\prime} \geq \gamma h^{2} k^{\prime}=\varepsilon,
$$

and cut-off function $x^{n} \varphi$ and with the aid of Lemma 4.6.

We have that

$$
\begin{aligned}
& \left|\sum_{k, k^{\prime} \geq n / 2} e^{i\left[t k k^{\prime}+\omega\left(k^{\prime}-k\right)\right]} \varphi\left(h^{2} k k^{\prime}\right) N k^{n-1} k^{\prime n-1} \sigma_{1}\left(k, k^{\prime}\right)\right| \\
& \leq \frac{C}{h^{2 n}} \sum_{k^{\prime}=n / 2}^{\left\lfloor c^{\prime} / h\right\rfloor} \frac{1}{k^{\prime}}\left|\sum_{k=n / 2}^{\left\lfloor c^{\prime} / h\right\rfloor} e^{i k\left[t k^{\prime}-\omega\right]} \varphi\left(h^{2} k k^{\prime}\right)\left(h^{2} k k^{\prime}\right)^{n} \sigma_{1}\left(k, k^{\prime}\right)\right| \\
& \quad+\frac{C}{h^{2 n-2}} \sum_{k^{\prime}=n / 2}^{\left\lfloor c^{\prime} / h\right\rfloor} k^{\prime}\left|\sum_{k=n / 2}^{\left\lfloor c^{\prime} / h\right\rfloor} e^{i k\left[t k^{\prime}-\omega\right]} \varphi\left(h^{2} k k^{\prime}\right)\left(h^{2} k k^{\prime}\right)^{n-1} \sigma_{1}\left(k, k^{\prime}\right)\right| .
\end{aligned}
$$


As in the previous case we may limit ourselves to consider the first sum in (4.25), the estimate for the latter one being analogous. We have

$$
\begin{aligned}
& \frac{1}{h^{2 n}} \sum_{k^{\prime}=1}^{\left\lfloor c^{\prime} / h\right\rfloor} \frac{1}{k^{\prime}}\left|\sum_{k=1}^{\left\lfloor c^{\prime} / h\right\rfloor} e^{i k\left[t k^{\prime}-\omega\right]} \varphi\left(h^{2} k k^{\prime}\right)\left(h^{2} k k^{\prime}\right)^{n} \sigma_{1}\left(k, k^{\prime}\right)\right| \\
& \quad \leq \frac{C}{h^{2 n}} \sum_{k^{\prime}=1}^{\left\lfloor c^{\prime} / h\right\rfloor+1} \frac{1}{k^{\prime}}\left(\frac{\left(h^{2} k^{\prime}\right)^{(L-1)}}{\left(t k^{\prime}\right)^{L}}+1\right) \\
& \leq \frac{C}{h^{2 n}}\left(\frac{h^{2(L-1)}}{t^{L}} \sum_{k^{\prime}=1}^{\left\lfloor c^{\prime} / h\right\rfloor} \frac{1}{k^{\prime 2}}+\log (1 / h)\right) \\
& \leq C\left(\frac{1}{t^{n+1}}+\frac{1}{h^{2(n+\kappa)}}\right),
\end{aligned}
$$

choosing $L=n+1$, for any $\kappa>0$.

Arguing as in (4.24) in Case (I), for $h^{2} \leq|t| \leq h^{s}$ we obtain

$$
\left|K_{p}^{1,+}(\omega, \theta)\right| \leq C \frac{1}{|t|^{n+1}} .
$$

The result now follows.

Step 5. Finally, we wish to estimate the modulus of $K_{2}^{ \pm}(\omega, \theta)$, as defined in (4.12). Again, we consider only the case of $K_{2}^{+}$.

In this case, it turns out that it suffices to take $m=0$. We introduce the same spectral decomposition as in (4.3). Thus, we are led to consider the remainder term

$$
\begin{aligned}
\left|K_{\mathcal{R}}^{2,+}(\omega, \theta)\right| & =\mid \sum_{k, k^{\prime} \geq n / 2} e^{i\left[t k k^{\prime}+\omega\left(k^{\prime}-k\right)\right]} \varphi\left(h^{2} k k^{\prime}\right) \tilde{\psi}\left(k, k^{\prime}\right) \eta_{+}\left(k^{\prime}-k\right) \chi_{2}(N \theta) \\
& \times N g_{n-1}\left(k^{\prime}\right) \tilde{g}_{n-1}(k) \theta^{m-n+2} \mathcal{R}_{m, N}(\theta) \mid \\
\leq & \frac{C}{h^{2 n-2}} \sum_{k=n / 2}^{\left\lfloor c^{\prime} / h\right\rfloor} \sum_{k^{\prime}=n / 2}^{\left\lfloor c^{\prime} / h\right\rfloor}\left|\varphi\left(h^{2} k k^{\prime}\right)\left(h^{2} k k^{\prime}\right)^{n-1} \frac{\theta^{m-n+2}}{N^{n+m-1 / 2}}\right| \\
\leq & \frac{C}{h^{2 n-2}} \sum_{k=n / 2}^{\left\lfloor c^{\prime} / h\right\rfloor} \sum_{k^{\prime}=n / 2}^{\left\lfloor c^{\prime} / h\right\rfloor} \frac{\theta^{m+3 / 2}}{(N \theta)^{n-1 / 2} N^{m}},
\end{aligned}
$$

so that, by choosing $m=0$, we obtain

$$
\left|K_{\mathcal{R}}^{2,+}(\omega, \theta)\right| \leq \frac{C}{h^{2 n}}
$$

for all $n \geq 1$, uniformly in $\omega$ and $\theta \in\left[0, \varepsilon_{1}\right]$.

Thus, for $j=2$ we are led to consider the main term in (4.12), that is,

$$
\Upsilon^{+}(\omega, \theta):=\sum_{k, k^{\prime} \geq n / 2} e^{i\left[t k k^{\prime}+\omega\left|k^{\prime}-k\right|\right]} \varphi\left(h^{2} k k^{\prime}\right) \tilde{\psi}\left(k, k^{\prime}\right) \eta_{+}\left(k^{\prime}-k\right) \chi_{2}(N \theta)
$$

$$
\times N g_{n-1}\left(k^{\prime}\right) \tilde{g}_{n-1}(k) \frac{J_{n-1}(N \theta)}{(N \theta)^{n-1}} .
$$


Recall that in this case we have that $N \theta \geq 1$. Then we use the asymptotic expansion of the Bessel function $J_{\nu}$

$$
J_{\nu}(x)=\frac{1}{x^{1 / 2}} \rho_{1}(x) e^{i x}+\frac{1}{x^{1 / 2}} \rho_{2}(x) e^{-i x}+\mathcal{O}\left(x^{-3 / 2}\right),
$$

for some bounded functions $\rho_{j}$, and write $\Upsilon^{+}(\omega, \theta)=\Upsilon_{1}+\Upsilon_{2}+\Upsilon_{3}$, where, for $j=1,2$,

$$
\begin{array}{r}
\Upsilon_{j}(\omega, \theta)=\sum_{k, k^{\prime} \geq n / 2} e^{i\left[t k k^{\prime}+\omega\left(k^{\prime}-k\right) \pm \theta\left(k+k^{\prime}\right)\right]} \varphi\left(h^{2} k k^{\prime}\right) \tilde{\psi}\left(k, k^{\prime}\right) \eta_{+}\left(k^{\prime}-k\right) \\
\times N g_{n-1}\left(k^{\prime}\right) \tilde{g}_{n-1}(k) \frac{1}{(N \theta)^{n-1 / 2}} \rho_{j}(N \theta) \chi_{2}(N \theta)
\end{array}
$$

and

$$
\begin{aligned}
\Upsilon_{3}(\omega, \theta)=\sum_{k, k^{\prime} \geq 1} e^{i\left[t k k^{\prime}+\omega\left(k^{\prime}-k\right)\right]} \varphi\left(h^{2} k k^{\prime}\right) \tilde{\psi}\left(k, k^{\prime}\right) \eta_{+}\left(k^{\prime}-k\right) \\
\quad \times N g_{n-1}\left(k^{\prime}\right) \tilde{g}_{n-1}(k) \frac{1}{(N \theta)^{n-1}} \chi_{2}(N \theta) \mathcal{O}\left((N \theta)^{-3 / 2}\right) .
\end{aligned}
$$

Lemma 4.9. For $N=k^{\prime}+k$, and $\beta=k^{\prime}-k$, set

$$
\sigma_{2}\left(k, k^{\prime}\right)=\tilde{\psi}\left(k, k^{\prime}\right) \eta_{+}\left(k^{\prime}-k\right) \frac{1}{(N \theta)^{n-\frac{1}{2}}} \chi_{2}(N \theta) R(N \theta),
$$

where

$$
R(N \theta)= \begin{cases}\rho_{j}(N \theta) & \text { for } \Upsilon_{j}, j=1,2 \\ (N \theta)^{1 / 2} \mathcal{O}\left((N \theta)^{-3 / 2}\right) & \text { for } \Upsilon_{3} .\end{cases}
$$

Then $\sigma_{2}$ is a symbol of order 0 in $k^{\prime}$ ( $k$ resp.), depending on the parameters $\theta$ and $k$ ( $k^{\prime}$ resp.), with norm uniformly bounded in such parameters.

Proof. As in Lemma 4.6 we wish to show that, considering $k^{\prime}=\xi$ as a continuous parameter, $\sigma_{2}$ is a smooth function of $\xi$ and, for each non-negative integer $m$, there exists a positive constant $C=C_{m}$, independent of $k$ and $\theta \in\left(0, \varepsilon_{1}\right)$, such that

$$
\left|\partial_{\xi}^{m} \sigma_{2}(\xi)\right| \leq C(1+|\xi|)^{-m}
$$

and we may assume that we have extended $\sigma_{2}$ to be identically 0 when $\xi \leq 1 / 2$.

Since $\chi_{2}(N \theta)=0$ for $N \theta \leq 1$ it follows that $\sigma_{2}$ is smooth and bounded as $\xi \rightarrow+\infty$, uniformly in $\theta$ and $k$.

Next we consider the derivatives. It the derivative falls on the factor $\frac{1}{(N \theta)^{n-\frac{1}{2}}}$ ( or on $\frac{1}{(N \theta)^{n-1}}$ in the case of $\Upsilon_{\mathcal{R}}$ ), using the condition $N \theta \geq 1$ we easily obtain, respectively, that

$$
\left|\partial_{\xi}\left[\frac{1}{(N \theta)^{n-\frac{1}{2}}}\right] \chi_{2}(N \theta) \tilde{\psi}\left(k, k^{\prime}\right) \eta_{+}\left(k^{\prime}-k\right)\right| \leq C \frac{1}{N} \leq C \frac{1}{\xi}
$$

and

$$
\left|\partial_{\xi}\left[\frac{1}{(N \theta)^{n-1}}\right] \chi_{2}(N \theta) \tilde{\psi}\left(k, k^{\prime}\right) \eta_{+}\left(k^{\prime}-k\right) R(N \theta)\right| \leq C \frac{1}{N} \leq C \frac{1}{\xi},
$$

as $\xi \rightarrow+\infty$, uniformly in $\theta$. 
If the derivative falls on $\rho_{j}(N \theta)$, by means of formula (15) in [St, p. 338] we observe that

$$
\left|\partial_{\xi}\left[\sum_{k} a_{k, j}(\xi \theta)^{-k}\right] \tilde{\psi}\left(k, k^{\prime}\right) \eta_{+}\left(k^{\prime}-k\right) \frac{1}{(N \theta)^{n-\frac{1}{2}}} \chi_{2}(N \theta)\right| \leq C \frac{1}{\xi},
$$

where $a_{k, j}, j=1,2$, are suitable coefficients.

If the derivative falls on $\chi_{2}$, we notice that $\chi_{2}^{\prime}(\xi)=0$ unless $1 \leq \xi \leq 2$, so that

$$
\left|\frac{1}{(N \theta)^{n-\frac{1}{2}}} \partial_{\xi}\left[\chi_{2}(N \theta)\right] \tilde{\psi}\left(k, k^{\prime}\right) \eta_{+}\left(k^{\prime}-k\right)\right| \leq C \theta \leq C \frac{1}{N} \leq C \frac{1}{\xi},
$$

and the same is true when $R(N \theta)$ is defined as in the latter case of (4.30).

Finally, if the derivative falls on the remainder term $\mathcal{O}\left((N \theta)^{-3 / 2}\right)$ in the latter case of (4.30), we have

$$
\left|\frac{1}{(N \theta)^{n-1}} \chi_{2}(N \theta) \tilde{\psi}\left(k, k^{\prime}\right) \eta_{+}\left(k^{\prime}-k\right) \partial_{\xi}\left[\mathcal{O}\left((N \theta)^{-3 / 2}\right)\right]\right| \leq C \frac{1}{N} \leq C \frac{1}{\xi} .
$$

Hence,

$$
\left|\partial_{\xi} \sigma_{2}(\xi)\right| \leq C(1+|\xi|)^{-1}
$$

as $|\xi| \rightarrow+\infty$, uniformly in $\theta$.

The argument can be repeated for all higher order derivatives. In particular, when the derivatives involve the term $\mathcal{O}\left((N \theta)^{-3 / 2}\right)$, we may use formula (8), p. 334 in [St, proving that this term behaves like a symbol of the expected order. Thus the lemma is proven.

In the case of $\Upsilon^{+}$we still need to use the oscillation of the phase and hence Lemma 4.4. Let $R_{I}, R_{I I}$ be as in Lemma 4.7. Since the phase in this case is $t k k^{\prime}+\omega\left(k^{\prime}-k\right) \pm \theta\left(k+k^{\prime}\right)$, and $\theta>0$, we write $\tilde{\theta}= \pm \theta$ and let $|\tilde{\theta}|$ vary in $\left[1 / N, \varepsilon_{1}\right]$. We then introduce the space of parameters $(t, \omega, \theta)$,

$$
R_{\theta}:=\left\{(t, \omega, \tilde{\theta}) \in\left[h^{2}, h^{s}\right] \times[0,2 \pi) \times\left(-\varepsilon_{1}, \varepsilon_{1}\right):|\tilde{\theta}|<t N / M_{1} \text { or }|\tilde{\theta}|>M_{1} N t\right\},
$$

where $M_{1}>2(1+M)$ is a large constant.

Lemma 4.10. Let $\theta$ be such that $1 \leq N|\tilde{\theta}|$. Let $\mathcal{V}_{+}$be defined as in (4.20). For $\left(k, k^{\prime}\right) \in \mathcal{V}_{+}$, set $\mu_{2}=(t k+\omega+\tilde{\theta}) / 2 \pi$ and $\mu_{2}^{\prime}=\left(t k^{\prime}-\omega+\tilde{\theta}\right) / 2 \pi$. Then, there exist a constant $\gamma>0$ and finitely many regions in $R_{\theta}$ such that for all $(t, \omega, \tilde{\theta})$ belonging to one of these regions, at least one between the two conditions

(III) $\operatorname{dist}\left(\mu_{2}, \mathbf{Z}\right) \geq \gamma t k$ for all $\left(k, k^{\prime}\right) \in \mathcal{V}_{+}$,

(IV) $\operatorname{dist}\left(\mu_{2}^{\prime}, \mathbf{Z}\right) \geq \gamma t k^{\prime}$ for all $\left(k, k^{\prime}\right) \in \mathcal{V}_{+}$

holds.

Proof. We begin observing that if either $|\tilde{\theta}|<t N / M_{1}$ or $|\tilde{\theta}|>M_{1} N t$, then there exists a constant $C>0$ such that

$$
|t k+\tilde{\theta}| \geq C t k \text { and }\left|t k^{\prime}+\tilde{\theta}\right| \geq C t k^{\prime} .
$$

Then we split the proof into a few cases.

Case 1. Let $R_{\theta, I}:=\left\{(t, \omega, \tilde{\theta}) \in R_{\theta}: 0<c_{1}<\omega<2 \pi-c_{1}\right\}$, where $c_{1}>10 \varepsilon_{1}$ is a small constant. Then (III) holds for $(t, \omega, \tilde{\theta}) \in R_{\theta, I}$ since

$\operatorname{dist}\left(\mu_{2}, \mathbf{Z}\right) \geq \operatorname{dist}(\omega / 2 \pi, \mathbf{Z})-\frac{t k}{2 \pi}-\frac{|\theta|}{2 \pi} \geq \frac{c_{1}}{2 \pi}-\frac{t k}{2 \pi}-\frac{\varepsilon_{1}}{2 \pi} \geq c_{1}\left(\frac{1}{2 \pi}-\frac{1}{20 \pi}\right)-\frac{t k}{2 \pi} \geq \gamma t k$, for all $k=1, \ldots, c^{\prime} / h$, as a consequence of (4.21). 
Hence, possibly replacing $\omega$ by $2 \pi-\omega$, we may assume that $|\omega| \leq c_{1}$, that $\operatorname{dist}\left(\mu_{2}, \mathbf{Z}\right)=|t k+\omega+\tilde{\theta}| / 2 \pi$ and that $\operatorname{dist}\left(\mu_{2}^{\prime}, \mathbf{Z}\right)=\left|t k^{\prime}-\omega+\tilde{\theta}\right| / 2 \pi$. Notice that now we may assume that $\tilde{\theta}<0$ since otherwise (III) holds on $R_{I}$ and (IV) on $R_{I I}$, where $R_{I}, R_{I I}$ are defined in Lemma 4.7

Case 2. Let $R_{\theta, I I}:=\left\{(t, \omega, \tilde{\theta}) \in R_{\theta}:|\omega|<c_{2} t\right\}$, where $c_{2}>0$ is a small constant. If $(t, \omega, \tilde{\theta}) \in R_{\theta, I I}$, then (III) holds for all $\left(k, k^{\prime}\right) \in \mathcal{V}_{+}$, since

$$
|t k+\omega+\tilde{\theta}| \geq|t k+\tilde{\theta}|-|\omega| \geq t\left(C k-c_{2}\right) \geq \gamma t k,
$$

provided that $c_{2}$ is small enough.

Case 3. Next suppose $c_{2} t \leq|\omega| \leq c_{1}$. If $\omega>0$, then, if $t k+\tilde{\theta}>0$, (III) holds. If $t k+\tilde{\theta}<0$, we first observe that $|\tilde{\theta}|>M_{1} t N$. Indeed, if $|\tilde{\theta}|<t N / M_{1}$, then

$$
|\tilde{\theta}|<\frac{1}{M_{1}} t\left(k+k^{\prime}\right) \leq \frac{1}{M_{1}} t k(1+M) \leq \frac{t k}{2},
$$

since we chose $M_{1}>2(1+M)$. Thus $t k+\tilde{\theta}>0$, contradicting the hypothesis.

Now it is easy to conclude that condition (IV) holds, since

$$
t k^{\prime}+\tilde{\theta}<t k^{\prime}-M_{1} t N<0,
$$

so that

$$
\left|t k^{\prime}+\tilde{\theta}-\omega\right|=\omega+\left|t k^{\prime}+\tilde{\theta}\right| \geq C t k^{\prime} .
$$

If $\omega<0$, then, if $t k+\tilde{\theta}<0$, (III) holds as a consequence of (4.31). If $t k+\tilde{\theta}>0$, we notice that $|\tilde{\theta}|<\frac{1}{M_{1}} t N$. Then condition (IV) holds, since

$$
t k^{\prime}+\tilde{\theta}>t k^{\prime}-\frac{1}{M_{1}} t N>t k^{\prime}\left(1-\frac{1}{M_{1}}\right)-\frac{M}{M_{1}} t k^{\prime}>0,
$$

provided that $M_{1}>1+M$, so that $\left|t k^{\prime}+\tilde{\theta}-\omega\right|=|\omega|+t k^{\prime}+\tilde{\theta} \geq C t k^{\prime}$.

Proposition 4.11. There exists a constant $C>0$ such that, for all $|t| \in A$ when $n>1$, and for all $h^{2} \leq|t| \leq C h^{4 / 3}$ when $n=1$,

$$
\left|\Upsilon_{j}(\omega, \theta)\right| \leq C \frac{1}{|t|^{n+1}}
$$

uniformly in $\omega$ and $\theta \in\left[0, \varepsilon_{1}\right]$, for $j=1,2$.

Proof. Recall that $\Upsilon_{j}$ have been defined in (4.29). Next, we fix a smooth cut-off function $\Psi$ with compact support in $\left[1 / M_{1}, M_{1}\right]$, where $M_{1}>1$ is a (large) constant. For $j=1,2$ we decompose $\Upsilon_{j}$ by setting

$$
\begin{aligned}
\Upsilon_{j}(\omega, \theta)= & \sum_{k, k^{\prime} \geq 1} e^{i\left[t k k^{\prime}+\omega\left(k^{\prime}-k\right) \pm \theta\left(k+k^{\prime}\right)\right]} \varphi\left(h^{2} k k^{\prime}\right) \tilde{\psi}\left(k, k^{\prime}\right) \eta_{+}\left(k^{\prime}-k\right) \\
& \times \chi_{2}(N \theta) \frac{N g_{n-1}\left(k^{\prime}\right) \tilde{g}_{n-1}(k)}{(N \theta)^{n-1 / 2}} \rho_{j}(N \theta)[1-\Psi(\theta / t N)] \\
& +\sum_{k, k^{\prime} \geq 1} e^{i\left[t k k^{\prime}+\omega\left(k^{\prime}-k\right) \pm \theta\left(k+k^{\prime}\right)\right]} \varphi\left(h^{2} k k^{\prime}\right) \tilde{\psi}\left(k, k^{\prime}\right) \eta_{+}\left(k^{\prime}-k\right) \\
& \times \chi_{2}(N \theta) \frac{N g_{n-1}\left(k^{\prime}\right) \tilde{g}_{n-1}(k)}{(N \theta)^{n-1 / 2}} \rho_{j}(N \theta) \Psi(\theta / t N) \\
(4.32)=: & \Upsilon_{j, 1}(\omega, \theta)+\Upsilon_{j, 2}(\omega, \theta),
\end{aligned}
$$


We also have

$$
\Upsilon_{3}(\omega, \theta)=\sum_{k, k^{\prime} \geq 1} e^{i\left[t k k^{\prime}+\omega\left(k^{\prime}-k\right)\right]} \varphi\left(h^{2} k k^{\prime}\right) N\left(k k^{\prime}\right)^{n-1} \sigma_{2}\left(k, k^{\prime}\right) .
$$

For $\Upsilon_{j, 1}$ we argue as in the proof of Proposition 4.8 and divide into the cases in which (III) or (IV) hold, respectively. If, for instance, (III) holds, then as in the proof of Case (I) in Proposition 4.8, we have

$$
\begin{aligned}
\left|\Upsilon_{j, 1}(\omega, \theta)\right| \leq & \frac{C}{h^{2 n-2}} \sum_{k=1}^{\left\lfloor c^{\prime} / h\right\rfloor}\left|\sum_{k^{\prime}=1}^{\left\lfloor c^{\prime} / h\right\rfloor} e^{i k^{\prime}[t k+\omega+\tilde{\theta}]} \varphi\left(h^{2} k k^{\prime}\right) N\left(h^{2} k k^{\prime}\right)^{n-1} \sigma_{2}(N)\right| \\
\leq & \frac{C}{h^{2 n}} \sum_{k=1}^{\left\lfloor c^{\prime} / h\right\rfloor} \frac{1}{k} \sum_{k^{\prime}=\left\lfloor a^{\prime} /\left(h^{2} k\right)\right\rfloor}^{\left\lfloor b^{\prime} /\left(h^{2} k\right)\right\rfloor+1} e^{i k^{\prime}[t k+\omega \pm \theta]} \varphi\left(h^{2} k k^{\prime}\right)\left(h^{2} k k^{\prime}\right)^{n} \sigma_{2}(N) \mid \\
& +\frac{C}{h^{2 n-2}} \sum_{k=1}^{\left\lfloor c^{\prime} / h\right\rfloor} \sum_{k^{\prime}=\left\lfloor a^{\prime} /\left(h^{2} k\right)\right\rfloor}^{\left\lfloor b^{\prime} /\left(h^{2} k\right)\right\rfloor+1} e^{i k^{\prime}[t k+\omega \pm \theta]} \varphi\left(h^{2} k k^{\prime}\right)\left(h^{2} k k^{\prime}\right)^{n-1} \sigma_{2}(N) \mid \\
\leq & \frac{C}{h^{2 n+\kappa}},
\end{aligned}
$$

for any $\kappa>0$, uniformly in $\omega$ and $\theta \in\left[0, \varepsilon_{1}\right]$, by proceeding as in the proof of (4.24) by means of Lemma 4.10. The proof in the case in which condition (IV) holds is analogous to the proof of (4.26) in Proposition 4.8, and it is omitted.

Finally we estimate $\Upsilon_{j, 2}$. In this sum we take advantage of the fact that $\theta / t N$ is bounded above and below from zero. We have

$$
\begin{aligned}
\left|\Upsilon_{j, 2}(\omega, \theta)\right| & \leq C \sum_{k, k^{\prime} \geq 1} \varphi\left(h^{2} k k^{\prime}\right) \tilde{\psi}\left(k, k^{\prime}\right) \eta_{+}\left(k^{\prime}-k\right) \chi_{2}(N \theta) \frac{N^{2 n-1}}{(N \theta)^{n-1 / 2}} \Psi(\theta / t N) \\
& \leq C \sum_{k, k^{\prime} \geq 1} \varphi\left(h^{2} k k^{\prime}\right) \tilde{\psi}\left(k, k^{\prime}\right) \eta_{+}\left(k^{\prime}-k\right) \frac{N^{2 n-1}}{\left(N^{2} t\right)^{n-1 / 2}} \\
& \leq \frac{C}{t^{n-1 / 2}} \sum_{k, k^{\prime} \geq 1} \varphi\left(h^{2} k k^{\prime}\right) \tilde{\psi}\left(k, k^{\prime}\right) \\
& \leq \frac{C}{t^{n-1 / 2} h^{2}} \leq \frac{C}{t^{n+1+1 / n-1 / 2}},
\end{aligned}
$$

since $t \leq h^{s}$, so that $h^{-2} \leq t^{-2 / s}<t^{-(n+1) / n}$. Thus when $n=1$

$$
\left|\Upsilon_{j, 2}(\omega, \theta)\right| \leq \frac{C}{t^{2}}
$$

for all $h^{2} \leq|t| \leq c h^{4 / 3}$, while if $n>1$

$$
\left|\Upsilon_{j, 2}(\omega, \theta)\right| \leq \frac{C}{t^{n+1}}
$$

for all $|t| \in A$. Finally, we observe that $\Upsilon_{3}$ may be treated as the sum in (4.22) by means of Lemma 4.7, so that

$$
\left|\Upsilon_{3}(\omega, \theta)\right| \leq C \frac{1}{|t|^{n+1}}
$$

uniformly in $\omega$ and $\theta \in\left[0, \varepsilon_{1}\right]$, for all $|t| \in\left[h^{2}, c h^{s}\right]$. 
Thus, as a consequence of the decompositions (4.14) and (4.28), of Propositions 4.8 and 4.11 by also using (4.16) and (4.27), if $n>1$ in (4.12) we obtain

$$
\left|K_{1}^{ \pm}(\omega, \theta)+K_{2}^{ \pm}(\omega, \theta)\right| \leq \frac{C}{|t|^{n+1}},
$$

uniformly in $\omega$ and $\theta \in\left[0, \pi / 2-\varepsilon_{1}\right]$, so that we finally get (4.2), that is,

$$
\sup _{(z, w) \in \Omega}\left|\sum_{\ell, \ell^{\prime}=1}^{+\infty} e^{i t \lambda_{\ell, \ell^{\prime}}} \varphi\left(h^{2} \lambda_{\ell, \ell^{\prime}}\right) \psi\left(\ell^{\prime} / \ell\right) Z_{\ell, \ell^{\prime}}(z, w)\right| \leq C \frac{1}{h^{2 n+\kappa}},
$$

for all $t \in A, n>1$. When $n=1$, as a consequence of Proposition 4.11 we get

$$
\sup _{(z, w) \in \Omega}\left|\sum_{\ell, \ell^{\prime}=1}^{+\infty} e^{i t \lambda_{\ell, \ell^{\prime}}} \varphi\left(h^{2} \lambda_{\ell, \ell^{\prime}}\right) \psi\left(\ell^{\prime} / \ell\right) Z_{\ell, \ell^{\prime}}(z, w)\right| \leq C \frac{1}{t^{2}}
$$

for all $|t| \in\left[h^{2}, c h^{4 / 3}\right]$.

This concludes the proof of Theorem 3.1 .

\section{The Strichartz estimate}

In this section we complete the proof of Theorem 1.1 .

Following a classical pattern we invoke a result by Keel and Tao [KT]. Consider the family of operators

$$
U(t):=\chi_{J}(t) e^{i t \mathcal{L}} \varphi\left(h^{2} \mathcal{L}\right),
$$

where $t \in \mathbf{R}, h \in(0,1], \chi_{J}$ denotes the characteristic function of the interval $J$ and $|J| \approx h^{\alpha}$, where, if $n>1$, we will select either $\alpha=s>s_{n}$ (with $s_{n}$ given by (1.6)) or $\alpha=2$. If $n=1$, we will select either $\alpha \geq 4 / 3$ or $\alpha=2$.

Then $U(t)$ satisfies the energy estimate $\|U(t)\|_{\left(L^{2}, L^{2}\right)} \leq C$ for some positive constant $C$ and the following untruncated decay estimate:

$$
\begin{gathered}
\left\|U(t) U(\tau)^{*} v_{0}\right\|_{L^{\infty}}\left\|\chi_{J}(t-\tau) e^{i(t-\tau) \mathcal{L}} \varphi\left(h^{2} \mathcal{L}\right) v_{0}\right\|_{L^{\infty}} \\
\leq \frac{C}{|t-\tau|^{Q / 2}}\left\|v_{0}\right\|_{L^{1}\left(S^{2 n+1}\right)}
\end{gathered}
$$

for all $t, \tau \in \mathbf{R}, t \neq \tau$. Hence Theorem 1.2 in $[\mathrm{KT}]$ yields the following result.

Proposition 5.1. For any fixed $\varphi \in \mathcal{C}_{0}^{\infty}\left(\mathbf{R}_{+}\right)$, there exists a constant $C>0$ such that for all $h \in(0,1]$, for any interval $J$ of length $|J| \leq h^{\alpha}$ and for all $v_{0} \in \mathcal{C}^{\infty}\left(S^{2 n+1}\right)$ the following estimate holds:

$$
\left(\int_{J}\left\|e^{i t \mathcal{L}} \varphi\left(h^{2} \mathcal{L}\right) v_{0}\right\|_{L^{q}}^{p} d t\right)^{1 / p} \leq C\left\|v_{0}\right\|_{L^{2}}
$$

for all pairs $(p, q) \neq(2,+\infty)$, satisfying (1.5), where $\alpha=2$ if $v_{0} \in \mathcal{C}_{\mathcal{E}}^{\infty}$ and $\alpha>s_{n}$ if $v_{0} \in \mathcal{C}_{\mathcal{V}}^{\infty}$, and $\mathcal{E}, \mathcal{V}$ are defined in (2.9) and (2.10). Here $C$ depends only on $p, q, n$ and $s$.

Finally, in order to prove the Strichartz estimate (1.7) we shall need an easy consequence of the Littlewood-Paley decomposition for the sublaplacian $\mathcal{L}$ on the complex sphere. More precisely, we shall use the following result. 
Theorem 5.2. Let $\tilde{\psi} \in \mathcal{C}_{0}^{\infty}\left(\mathbf{R}_{+}\right)$and $\psi \in \mathcal{C}_{0}^{\infty}(\mathbf{R})$ such that

$$
\tilde{\psi}(\lambda)+\sum_{j=1}^{\infty} \psi\left(2^{-2 j} \lambda\right)=1, \lambda \in \mathbf{R} .
$$

Then for $2 \leq q<\infty$ there exists a constant $C_{q}$ such that

$$
\|f\|_{L^{q}\left(S^{2 n+1}\right)} \leq C_{q}\left(\|\tilde{\psi}(\mathcal{L}) f\|_{L^{q}\left(S^{2 n+1}\right)}+\left(\sum_{j=1}^{+\infty}\left\|\psi\left(2^{-2 j} \mathcal{L}\right) f\right\|_{L^{q}\left(S^{2 n+1}\right)}^{2}\right)^{1 / 2}\right),
$$

for $f \in L^{q}\left(S^{2 n+1}\right)$.

This result and the Littlewood-Paley decomposition are of independent interest (see the recent paper Bouc for a discussion of analogous inequalities in the Riemannian case) and may be easily deduced from the multiplier theorem in CowKS.

End of the proof of Theorem 1.1, Let $v_{0} \in \mathcal{C}_{\mathcal{V}}^{\infty}, v_{0} \in \mathcal{C}_{\mathcal{E}}^{\infty}$ respectively, and $\alpha=s>$ $s_{n}, \alpha=2$ respectively.

By writing $[-1,1]=\bigcup_{k=1}^{N} J_{k}$, with $J_{k}$ intervals, $\left|J_{k}\right| \approx h^{\alpha}$ and $N \approx h^{-\alpha}$, we have

$$
\begin{aligned}
\int_{-1}^{1}\left\|e^{i t \mathcal{L}} \varphi\left(h^{2} \mathcal{L}\right) v_{0}\right\|_{L^{q}}^{p} d t & \leq \sum_{k=1}^{N} \int_{J_{k}}\left\|e^{i t \mathcal{L}} \varphi\left(h^{2} \mathcal{L}\right) v_{0}\right\|_{L^{q}}^{p} d t \\
& \leq C N\left\|v_{0}\right\|_{L^{2}}^{p} \\
& \leq C h^{-\alpha}\left\|v_{0}\right\|_{L^{2}}^{p}
\end{aligned}
$$

so that

$$
\left(\int_{-1}^{1}\left\|e^{i t \mathcal{L}} \varphi\left(h^{2} \mathcal{L}\right) v_{0}\right\|_{L^{q}}^{p} d t\right)^{1 / p} \leq C h^{-\alpha / p}\left\|v_{0}\right\|_{L^{2}} .
$$

Now, let $\tilde{\varphi} \in \mathcal{C}_{0}^{\infty}\left(\mathbf{R}_{+}\right)$be such that $\tilde{\varphi} \varphi=\varphi$. Then (5.4), with $\varphi$ replaced by $\tilde{\varphi}$ and the initial datum $\varphi\left(h^{2} \mathcal{L}\right) v_{0}$, gives

$$
\left(\int_{-1}^{1}\left\|e^{i t \mathcal{L}} \tilde{\varphi}\left(h^{2} \mathcal{L}\right) \varphi\left(h^{2} \mathcal{L}\right) v_{0}\right\|_{L^{q}}^{p} d t\right)^{1 / p} \leq C h^{-\alpha / p}\left\|\varphi\left(h^{2} \mathcal{L}\right) v_{0}\right\|_{L^{2}}
$$

that is,

$$
\left(\int_{-1}^{1}\left\|e^{i t \mathcal{L}} \varphi\left(h^{2} \mathcal{L}\right) v_{0}\right\|_{L^{q}}^{p} d t\right)^{1 / p} \leq C h^{-\alpha / p}\left\|\varphi\left(h^{2} \mathcal{L}\right) v_{0}\right\|_{L^{2}}
$$

We now apply Theorem 5.2 to $f=v(t)=e^{i t \mathcal{L}} v_{0}$ and then we take the $L^{p}$-norm with respect to the variable $t$ on $[-1,1]$ and obtain that

$$
\begin{aligned}
& \|v\|_{L^{p}\left([-1,1], L^{q}\left(S^{2 n+1}\right)\right)} \\
& \quad \leq C\left(\left\|v_{0}\right\|_{L^{2}\left(S^{2 n+1}\right)}+\left\|\left(\sum_{j=1}^{+\infty}\left\|e^{i t \mathcal{L}} \psi\left(2^{-2 j} \mathcal{L}\right) v_{0}\right\|_{L^{q}\left(S^{2 n+1}\right)}^{2}\right)^{1 / 2}\right\|_{L^{p}([-1,1])}\right) .
\end{aligned}
$$


Next, let $v_{0}$ be any function in $\mathcal{C}^{\infty}\left(S^{2 n+1}\right)$. Since $p \geq 2$, by Minkowski's integral inequality we have

$$
\begin{aligned}
& \left\|\left(\sum_{j=1}^{+\infty}\left\|e^{i t \mathcal{L}} \psi\left(2^{-2 j} \mathcal{L}\right) v_{0}\right\|_{L^{q}\left(S^{2 n+1}\right)}^{2}\right)^{1 / 2}\right\|_{L^{p}([-1,1])} \\
& \leq\left(\sum_{j=1}^{+\infty}\left(\int_{-1}^{1}\left\|e^{i t \mathcal{L}} \psi\left(2^{-2 j} \mathcal{L}\right) v_{0}\right\|_{L^{q}\left(S^{2 n+1}\right)}^{p} d t\right)^{2 / p}\right)^{1 / 2} \\
& \leq\left(\sum_{j=1}^{+\infty}\left(\int_{-1}^{1}\left\|e^{i t \mathcal{L}} \psi\left(2^{-2 j} \mathcal{L}\right) \pi_{\mathcal{V}} v_{0}\right\|_{L^{q}\left(S^{2 n+1}\right)}^{p} d t\right)^{2 / p}\right)^{1 / 2} \\
& \quad+\left(\sum_{j=1}^{+\infty}\left(\int_{-1}^{1}\left\|e^{i t \mathcal{L}} \psi\left(2^{-2 j} \mathcal{L}\right) \pi_{\mathcal{E}} v_{0}\right\|_{L^{q}\left(S^{2 n+1}\right)}^{p} d t\right)^{2 / p}\right)^{1 / 2} \\
& \leq C\left(\sum_{j=1}^{+\infty} 2^{(2 j s / p)}\left\|\psi\left(2^{-2 j} \mathcal{L}\right) \pi_{\mathcal{V}} v_{0}\right\|_{L^{2}\left(S^{2 n+1}\right)}^{2}\right)^{1 / 2} \\
& \quad+C\left(\sum_{j=1}^{+\infty} 2^{(4 j / p)}\left\|\psi\left(2^{-2 j} \mathcal{L}\right) \pi_{\mathcal{E}} v_{0}\right\|_{L^{2}\left(S^{2 n+1}\right)}^{2}\right)^{1 / 2} \\
& \leq C\left\|v_{0}\right\|_{\mathcal{X}(s / p, 2 / p)}
\end{aligned}
$$

where we used, in particular, the Strichartz estimate (5.5) for the spectral truncations. This yields (1.7). Analogous arguments lead to

$$
\left\|\left(\sum_{j=1}^{+\infty}\left\|e^{i t \mathcal{L}} \psi\left(2^{-2 j} \mathcal{L}\right) v_{0}\right\|_{L^{q}\left(S^{3}\right)}^{2}\right)^{1 / 2}\right\|_{L^{p}([-1,1])} \leq C\left\|v_{0}\right\|_{\mathcal{X}^{(s / p, 2 / p)}}
$$

for all $s \geq 4 / 3$, when $n=1$.

The following result follows at once from the Strichartz estimates (1.7) and the Minkowski inequality.

Corollary 5.3. If $p$ and $q$ satisfy $\frac{2}{p}+\frac{Q}{q}=\frac{Q}{2}, p \geq 2, q<\infty$, then for all $T>0$ and for all $s>s_{n}, s_{n}$ defined by (1.6), if $n>1$, or for all $s \geq 4 / 3$ if $n=1$, there exists $C=C(p, T, s)$ such that for every $f \in L^{1}\left([-T, T], \mathcal{X}^{s / p, 2 / p}\right)$ we have

$$
\left\|\int_{0}^{t} e^{i\left(t-t^{\prime}\right) \mathcal{L}} f\left(t^{\prime}\right) d t^{\prime}\right\|_{L^{p}\left([-T, T], L^{q}\left(S^{2 n+1}\right)\right)} \leq C\|f\|_{L^{1}\left([-T, T], \mathcal{X}^{(s / p, 2 / p)}\right)} .
$$

\section{FinAL REMARKS}

6.1. Discussion of optimality. Strichartz estimates proved in Theorem 1.1 are, in general, not sharp. To study optimality, we may use some sharp estimates for the joint spectral projections $\pi_{\ell, \ell^{\prime}}$, proved by the first author in [Ca1, Ca2.

More precisely, consider an eigenfunction of the sublaplacian $\mathcal{L}$ corresponding to the eigenvalue $N=\lambda_{\ell, \ell^{\prime}}$, and then take the solution of the homogeneous Schrödinger equation $v(t, z)=e^{-i t \lambda_{\ell, \ell^{\prime}}} v_{0}$, with initial datum $v_{0}=h_{\ell, \ell^{\prime}}$. Here $h_{\ell, \ell^{\prime}}$ is a spherical harmonic in $\mathcal{H}^{\ell, \ell^{\prime}}$ such that

$$
\left\|h_{\ell, \ell^{\prime}}\right\|_{L^{q}\left(S^{2 n+1}\right)} \geq \frac{1}{C}\left(\lambda_{\ell, \ell^{\prime}}+1\right)^{\alpha(1 / q, n)}\left(\ell+\ell^{\prime}+1\right)^{\beta(1 / q, n)}\left\|h_{\ell, \ell^{\prime}}\right\|_{L^{2}\left(S^{2 n+1}\right)},
$$


where

$$
\alpha(1 / q, n)=\left\{\begin{array}{lll}
n\left(\frac{1}{2}-\frac{1}{q}\right)-\frac{1}{2} & \text { if } & q \geq 2 \frac{2 n+1}{2 n-1} \\
\frac{1}{2}\left(\frac{1}{q}-\frac{1}{2}\right) & \text { if } & 2 \leq q \leq 2 \frac{2 n+1}{2 n-1}
\end{array}\right.
$$

and

$$
\beta(1 / q, n)=\left\{\begin{array}{lll}
\frac{1}{2} & \text { if } & q \geq 2 \frac{2 n+1}{2 n-1}, \\
\left(n+\frac{1}{2}\right)\left(\frac{1}{2}-\frac{1}{q}\right) & \text { if } & 2 \leq q \leq 2 \frac{2 n+1}{2 n-1} .
\end{array}\right.
$$

We refer to Theorem 3.1 and Proposition 3.4 in [Ca2 for the details.

Then we have

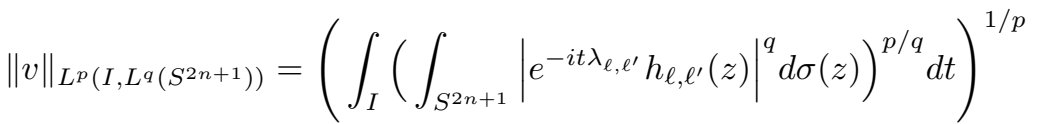

$$
\begin{aligned}
& =\ell(I)^{1 / p}\left\|h_{\ell, \ell^{\prime}}\right\|_{L^{q}\left(S^{2 n+1}\right)} \\
& \geq \frac{1}{C}\left(\lambda_{\ell, \ell^{\prime}}+1\right)^{\alpha(1 / q, n)}\left(\ell+\ell^{\prime}+1\right)^{\beta(1 / q, n)}\left\|h_{\ell, \ell^{\prime}}\right\|_{L^{2}\left(S^{2 n+1}\right)} \text {. }
\end{aligned}
$$

Now if $\left(\ell, \ell^{\prime}\right) \in \mathcal{V}$, for some fixed proper cone $\mathcal{V}$, defined as in (2.9), and if $p, q$ satisfy the admissibility condition (1.5), then

$$
\|v\|_{L^{p}\left(I, L^{q}\left(S^{2 n+1}\right)\right)} \geq \frac{1}{C}\left(\lambda_{\ell, \ell^{\prime}}+1\right)^{\alpha+\beta / 2}\left\|h_{\ell, \ell^{\prime}}\right\|_{L^{2}\left(S^{2 n+1}\right)} \approx\left\|v_{0}\right\|_{W^{\alpha+\beta / 2}\left(S^{2 n+1}\right)} .
$$

It is easy to check that $s / p>s_{n} / p>\alpha+\beta / 2$ so that this estimate does not provide the sharp bound.

If $\left(\ell, \ell^{\prime}\right) \in \mathcal{E}$, where $\mathcal{E}$ is defined as in (2.10), then

$$
\begin{aligned}
\|v\|_{L^{p}\left(I, L^{q}\left(S^{2 n+1}\right)\right)} & \geq \frac{1}{C}\left(\lambda_{\ell, \ell^{\prime}}+1\right)^{\alpha+\beta}\left\|h_{\ell, \ell^{\prime}}\right\|_{L^{2}\left(S^{2 n+1}\right)} \\
& \geq \frac{1}{C}\left(\lambda_{\ell, \ell^{\prime}}+1\right)^{\frac{2 n}{p Q}}\left\|h_{\ell, \ell^{\prime}}\right\|_{L^{2}\left(S^{2 n+1}\right)} \approx \frac{1}{C}\left\|v_{0}\right\|_{W^{4 n / p Q}\left(S^{2 n+1}\right)} .
\end{aligned}
$$

Now observe that

$$
\frac{4 n}{p Q} \frac{2}{p}\left(1-\frac{1}{n+1}\right)=s_{n}, \quad \text { for } n>1
$$

so that

$$
\|v\|_{L^{p}\left(I, L^{q}\left(S^{2 n+1}\right)\right)} \geq \frac{1}{C}\left\|v_{0}\right\|_{W^{\frac{2}{p}(1-1 /(n+1))}}
$$

for all $(p, q)$ satisfying (1.5). Anyway, in Theorem 1.1 we proved that if $\left(\ell, \ell^{\prime}\right) \in \mathcal{E}$, then the critical index is $2 / p$, instead of $s_{n}$. In other words, the index $s>\frac{2}{p}\left[1-\frac{1}{n+1}\right]$ in Theorem 1.1 would be sharp, up to the loss of $\varepsilon$ derivatives, if we were able to prove an estimate like (1.7) with the space $\mathcal{X}^{(s / p, 2 / p)}\left(S^{2 n+1}\right)$ replaced by $W^{s / p}$.

6.2. Comparison with other subriemannian frameworks. As recalled in the Introduction, it has been proved in $\mathrm{BaGX}$ that no (global in time) dispersive estimate may hold for solutions of the Schrödinger equation on $\mathbf{H}_{n}$. Anyway, the situation seems to be less rigid on the reduced Heisenberg group $\mathbf{h}_{n}$, defined as $\mathbf{h}_{n}: \mathbf{C}^{n} \times \mathbf{T}$, with product

$$
\left(z, e^{i t}\right)\left(w, e^{i t^{\prime}}\right):=\left(z+w, e^{i\left(t+t^{\prime}+\Im m z \bar{w}\right)}\right),
$$

with $z, w \in \mathbf{C}^{n}, t, s \in \mathbf{R}$, due to the compacteness of the center. We point out that there is an intimate connection between the reduced Heisenberg group and the unit 
complex sphere, since $\mathbf{h}_{n}$ turns out to be a contraction of $S^{2 n+1}$ (see [CaCi] for more details about the construction of this contractive map). A detailed discussion of (local in time) dispersive estimates and of Strichartz estimates for solutions of the Schrödinger equation on $\mathbf{h}_{n}$ requires some additional care and will be presented elsewhere.

6.3. Discussion of the admissibility conditions. Admissibility condition (1.5) has been directly inspired by the scale invariance condition (1.10) on a Riemannian compact manifold of dimension $d$ (which in turn has been inherited by the euclidean space $\mathbf{R}^{d}$ ), with the dimension $d$ replaced by the homogeneous dimension $Q$. Anyway, on the CR sphere the notion of dilation, which leads to (1.10) in the euclidean context, is not intrinsic. An interesting possibility could be investigating scaling conditions in the subriemannian framework of the reduced Heisenberg group, where dilations are well defined as $\lambda \circ(z, t)=\left(\lambda z_{1}, \ldots, \lambda z_{n}, \lambda^{2} t\right)$, and then importing them on $S^{2 n+1}$.

\section{REFERENCES}

[BaCh] Hajer Bahouri and Jean-Yves Chemin, Équations d'ondes quasilinéaires et estimations de Strichartz (French, with French summary), Amer. J. Math. 121 (1999), no. 6, 13371377. MR 1719798 (2000i:35124)

[BaGX] Hajer Bahouri, Patrick Gérard, and Chao-Jiang Xu, Espaces de Besov et estimations de Strichartz généralisées sur le groupe de Heisenberg (French, with English summary), J. Anal. Math. 82 (2000), 93-118, DOI 10.1007/BF02791223. MR1799659 (2001k:58054)

[BauW] F. Baudoin and J. Wang, The Subelliptic Heat Kernel on the CR sphere, Math. Z. 275 (2013), no. 1-2, 135-150. MR3101801

[BlSSo1] Matthew D. Blair, Hart F. Smith, and Christopher D. Sogge, On Strichartz estimates for Schrödinger operators in compact manifolds with boundary, Proc. Amer. Math. Soc. 136 (2008), no. 1, 247-256 (electronic), DOI 10.1090/S0002-9939-07-09114-9. MR 2350410 (2008k:35386)

[BlSSo2] Matthew D. Blair, Hart F. Smith, and Christopher D. Sogge, Strichartz estimates and the nonlinear Schrödinger equation on manifolds with boundary, Math. Ann. 354 (2012), 1397-1430. MR2993000

[BoCl] Aline Bonami and Jean-Louis Clerc, Sommes de Cesàro et multiplicateurs des développements en harmoniques sphériques (French), Trans. Amer. Math. Soc. 183 (1973), 223-263. MR0338697 (49 \#3461)

[Bouc] Jean-Marc Bouclet, Littlewood-Paley decompositions on manifolds with ends (English, with English and French summaries), Bull. Soc. Math. France 138 (2010), no. 1, 1-37. MR2638890 (2011e:42020)

[Bou1] J. Bourgain, Fourier transform restriction phenomena for certain lattice subsets and applications to nonlinear evolution equations. I. Schrödinger equations, Geom. Funct. Anal. 3 (1993), no. 2, 107-156, DOI 10.1007/BF01896020. MR.1209299 (95d:35160a)

[Bou2] J. Bourgain, Exponential sums and nonlinear Schrödinger equations, Geom. and Funct. Anal. 3 (1993), 157-178. MR.1209300 (95d:35159)

[BrFM] T. Branson, L. Fontana and C. Morpurgo, Moser-Trudinger and Beckner-Onofri's inequalities on the CR sphere, Ann. Math. (2) 177 (2013), no. 1, 1-52. MR2999037

[BuGT1] Nicolas Burq, Patrick Gérard, and Nikolay Tzvetkov, The Schrödinger equation on a compact manifold: Strichartz estimates and applications (English, with English and French summaries), (Plestin-les-Grèves, 2001), Univ. Nantes, Nantes, 2001, pp. Exp. No. V, 18. MR 1843406 (2003a:58055)

[BuGT2] N. Burq, P. Gérard, and N. Tzvetkov, Strichartz inequalities and the nonlinear Schrödinger equation on compact manifolds, Amer. J. Math. 126 (2004), no. 3, 569-605. MR2058384 (2005h:58036) 
[BuGT3] N. Burq, P. Gérard, and N. Tzvetkov, Bilinear eigenfunction estimates and the nonlinear Schrödinger equation on surfaces (English, with English and French summaries), Invent. Math. 159 (2005), no. 1, 187-223, DOI 10.1007/s00222-004-0388-x. MR2142336 (2005m:35275)

[BuGT4] Nicolas Burq, Patrick Gérard, and Nikolay Tzvetkov, Multilinear eigenfunction estimates and global existence for the three dimensional nonlinear Schrödinger equations (English, with English and French summaries), Ann. Sci. École Norm. Sup. (4) 38 (2005), no. 2, 255-301, DOI 10.1016/j.ansens.2004.11.003. MR.2144988 (2006m:35337)

[Ca1] Valentina Casarino, Norms of complex harmonic projection operators, Canad. J. Math. 55 (2003), no. 6, 1134-1154, DOI 10.4153/CJM-2003-045-6. MR2016243 (2004m:43013)

[Ca2] Valentina Casarino, Two-parameter estimates for joint spectral projections on complex spheres, Math. Z. 261 (2009), no. 2, 245-259, DOI 10.1007/s00209-008-0323-8. MR2457298 (2009k:43012)

[CaCi] Valentina Casarino and Paolo Ciatti, Transferring $L^{p}$ eigenfunction bounds from $S^{2 n+1}$ to $h^{n}$, Studia Math. 194 (2009), no. 1, 23-42, DOI 10.4064/sm194-1-2. MR2520038 (2010f:43015)

[CaP1] Valentina Casarino and Marco M. Peloso, $L^{p}$-summability of Riesz means for the sublaplacian on complex spheres, J. Lond. Math. Soc. (2) 83 (2011), no. 1, 137-152, DOI 10.1112/jlms/jdq067. MR2763948 (2011k:42023)

[CazWe] Thierry Cazenave and Fred B. Weissler, The Cauchy problem for the critical nonlinear Schrödinger equation in $H^{s}$, Nonlinear Anal. 14 (1990), no. 10, 807-836, DOI 10.1016/0362-546X(90)90023-A. MR 1055532 (91j:35252)

$[\mathrm{ChX}]$ Jean-Yves Chemin and Chao-Jian $\mathrm{Xu}$, Inclusions de Sobolev en calcul de WeylHörmander et champs de vecteurs sous-elliptiques (French, with English and French summaries), Ann. Sci. École Norm. Sup. (4) 30 (1997), no. 6, 719-751, DOI 10.1016/S0012-9593(97)89937-5. MR1476294(98i:35026)

[CowKS] Michael G. Cowling, Oldrich Klima, and Adam Sikora, Spectral multipliers for the Kohn sublaplacian on the sphere in $\mathbb{C}^{n}$, Trans. Amer. Math. Soc. 363 (2011), no. 2, 611-631, DOI 10.1090/S0002-9947-2010-04920-7. MR2728580(2011i:42018)

$[\mathrm{FiH}] \quad$ A. Fitouhi and M. M. Hamza, A uniform expansion for the eigenfunction of a singular second-order differential operator, SIAM J. Math. Anal. 21 (1990), no. 6, 1619-1632, DOI 10.1137/0521088. MR1075594 (92a:33006)

[Fo1] G. B. Folland, The tangential Cauchy-Riemann complex on spheres, Trans. Amer. Math. Soc. 171 (1972), 83-133. MR0309156 (46 \#8266)

[Fo2] G. B. Folland, Subelliptic estimates and function spaces on nilpotent Lie groups, Ark. Mat. 13 (1975), no. 2, 161-207. MR0494315 (58 \#13215)

[Fo3] G. B. Folland, Spherical harmonic expansion of the Poisson-Szegö kernel for the ball, Proc. Amer. Math. Soc. 47 (1975), 401-408. MR0370044 (51 \#6273)

[FoSt] G. B. Folland and E. M. Stein, Estimates for the $\partial_{b}$ complex and analysis on the Heisenberg group, Comm. Pure Appl. Math. 27 (1974), 429-522. MR0367477 (51 \#3719)

[Ge] Daryl Geller, The Laplacian and the Kohn Laplacian for the sphere, J. Differential Geom. 15 (1980), no. 3, 417-435 (1981). MR620896 (82i:35132)

[GG1] Patrick Gérard and Sandrine Grellier, The cubic Szegő equation (English, with English and French summaries), Ann. Sci. Éc. Norm. Supér. (4) 43 (2010), no. 5, 761-810. MR2721876 (2012b:37188)

[GG2] Patrick Gérard and Sandrine Grellier, Invariant tori for the cubic Szegö equation, Invent. Math. 187 (2012), no. 3, 707-754. MR2944951

[GiV] J. Ginibre and G. Velo, Generalized Strichartz inequalities for the wave equation, J. Funct. Anal. 133 (1995), no. 1, 50-68, DOI 10.1006/jfan.1995.1119. MR.1351643 (97a:46047)

[JeLee] David Jerison and John M. Lee, The Yamabe problem on CR manifolds, J. Differential Geom. 25 (1987), no. 2, 167-197. MR880182 (88i:58162)

[KT] Markus Keel and Terence Tao, Endpoint Strichartz estimates, Amer. J. Math. 120 (1998), no. 5, 955-980. MR1646048(2000d:35018)

[Lee] John M. Lee, The Fefferman metric and pseudo-Hermitian invariants, Trans. Amer. Math. Soc. 296 (1986), no. 1, 411-429, DOI 10.2307/2000582. MR837820 (87j:32063)

[MPR] D. Müller, M. M. Peloso, and F. Ricci, Analysis of the Hodge Laplacian on the Heisenberg group, Mem. Amer. Math. Soc., to appear, ArXiv:1206.4540. 
[Na] A. Nagel, Introduction to analysis on Carnot-Carathéodory spaces, Notes for the Summer School in Harmonic Analysis, Centro di Ricerca Matematica Ennio De Giorgi, Pisa (2003), available on www.math.wisc.edu/ nagel/Combined.pdf.

[RU] Fulvio Ricci and Jérémie Unterberger, Solvability of invariant sublaplacians on spheres and group contraction (English, with English and Italian summaries), Atti Accad. Naz. Lincei Cl. Sci. Fis. Mat. Natur. Rend. Lincei (9) Mat. Appl. 12 (2001), 27-42 (2002). MR:1898446 (2003f:35052)

[St] Elias M. Stein, Harmonic analysis: real-variable methods, orthogonality, and oscillatory integrals, Princeton Mathematical Series, vol. 43, Princeton University Press, Princeton, NJ, 1993. With the assistance of Timothy S. Murphy; Monographs in Harmonic Analysis, III. MR $1232192(95 \mathrm{c}: 42002)$

[Str] Robert S. Strichartz, Restrictions of Fourier transforms to quadratic surfaces and decay of solutions of wave equations, Duke Math. J. 44 (1977), no. 3, 705-714. MR.0512086 (58 \#23577)

[Sz] G. Szegö, Orthogonal Polynomials, Colloquium Publications, American Mathematical Society, New York, 1939. MR0000077

[Tat] Daniel Tataru, Strichartz estimates for operators with nonsmooth coefficients and the nonlinear wave equation, Amer. J. Math. 122 (2000), no. 2, 349-376. MR.1749052 (2001c:35260)

[ViK] N. Ja. Vilenkin and A. U. Klymik, Representation of Lie groups and special functions, Vol. 2, Kluwer Academic Publishers, 1993. MR,1220225 ITALY

DTG, Università degli Studi di Padova, Stradella san Nicola 3, I-36100 Vicenza,

E-mail address: valentina.casarino@unipd.it

Dipartimento di Matematica, Università degli Studi di Milano, Via C. Saldini 50, I-20133 MiLAno, ItALy

E-mail address: marco.peloso@unimi.it 